REVISTA PROYECCIONES: 109-150.

JORNADAS MATEMATICAS, AGOSTO 1983.

\title{
BASES DE LA TEORÍA CUALITATIVA DE LAS ECUACIONES
}

\author{
DI FERENCI ALES
}

Dr. JORGE BILLEKE GONZALEZ*

§. ०. INTRODUCCION.

Este cursillo reúne el material básico para una introducción a la Teoría Cualitativa de las Ecuaciones Diferenciales.

Todo lo que se desarrolla está en el excelente libro de Jorge Sotomayor "Liçóes de Equiąóes Diferenciais Ordinárias" Projecto Eu clides, IMPA, Rio de Janeiro.

El tratamiento de esta introducción es totalmente auto-sufi ciente, basta un curso de Cálculo de Ecuaciones Diferenciales. Merece una especial atención la construcción de los Teoremas de Existencia y diferenciabilidad usando el Teorema de contracción en las Fibras.

* Profesor Departamento de Matemática y Ciencia de la Computación, Facultad de Ciencia. Universidad de Santiago de Chile. 
El objetivo es estudiar sistemas de ecuaciones diferenciales au tónomas de la forma

(1)

$$
\left\{\begin{array}{c}
x_{1}^{\prime}=x_{1}\left(x_{1}, \ldots, x_{n}\right) \\
x_{2}^{\prime}=x_{2}\left(x_{1}, \ldots, x_{n}\right) \\
\vdots \\
x_{n}^{\prime}=x_{n}\left(x_{1}, \ldots, x_{n}\right)
\end{array}\right.
$$

Nos interesa el análisis del retrato de Fase, es decir la parti ción del dominio de (1), que es un abierto de $\mathbb{R}^{n}$ por las curvas integra les del sistema.

El primero en abordar este problema fue H. Poincaré en su estudio de la estabilidad del sistema solar.

Dos cuestiones son interesantes a investigar: Ubicación y distribución de puntos singulares y órbitas periódicas, comportamientos asin tóticos de las curvas integrales y 10 que se llama Estabilidad estructural del sistema (Persistencia de la configuración topológica del retrato de Fase por pequeñas perturbaciones del sistema).

Aquí solo daremos las definiciones relevantes y las herramientas básicas para el análisis cualitativo.

Un texto introductorio pero completo es "Sistemas Dinámicos Diferenciables", Notas del seminario Interuniversitario de sistemas dinámi cos, USACH, 1983.

\section{§.1. CAMPOS VECTORTALES $\mathbf{Y}$ FLUJOS.}

1.1. Sea $U \subset \mathbb{R}^{n}$ abierto. Un Campo vectorial de Clase $C^{k} I \leqslant k \leqslant \infty$ es una aplicación $\mathrm{X}: U \rightarrow \mathbb{R}^{\mathrm{n}}$ de clase $C^{\mathrm{k}}$. A todo Campo, se le puede asociar una ecuación diferencial

$$
x^{\prime}=x(x)
$$


Las soluciones a esta ecuación, que son, $\rho: I \mathrm{c} \mathbb{R} \rightarrow U$, $\rho$ diferenciable tal que $\left.\frac{d \rho}{d t}(t)=x(\rho / t)\right) \forall t \in I$ se llaman Trayectorias - Curvas integrales de $\mathrm{X}$.

1.2. $x_{0} \in U$ se dice una Singularidad de $x$, $\circ$ un punto singular de $x \quad \circ$ un punto de equilibrio de $x$ si $x\left(x_{0}\right)=0$.

$\mathrm{x}_{0} \in \mathrm{U}$ se dice un punto regular si $\mathrm{x}\left(\mathrm{x}_{0}\right) \neq 0$

1.3. Lema: Si $x_{0}$ es punto singular, entonces $\rho(t)=x_{0},-\infty<t<\infty$ es solución de $x^{\prime}=x(x)$

Recíprocamente si $\rho(t)=x_{0^{\prime}}-\infty<t<\infty$ es solución de $x^{\prime}=x(x)$, entonces $\mathrm{x}_{0}$ es punto singular de $\mathrm{x}$.

Demostración:

$$
0=\rho^{\prime}(t)=x(\rho(t))=x\left(x_{0}\right)
$$

1.4. Una curva integral $\rho: I \rightarrow U$ de $X$ se llama máxima si para toda curva integral $\psi: J \rightarrow U$ con $I \subseteq J y \rho=\left.\psi\right|_{I}$ se tiene $I=J$ I se llama en este caso intervalo máximo.

\subsection{Teorema:}

a) Existencia y unicidad de soluciones máximas.

$\forall \mathrm{x} \in \mathrm{U}$ existe un intervalo abierto $I_{\mathrm{x}}$ donde está definida una única solución máxima $\rho_{x}$ de $x^{\prime}=x(x)$ tal que $\rho_{x}(0)=x$

b) Propiedad de grupo.

$$
\begin{aligned}
& \quad \text { Si } y=\rho_{x}(t), t \in I_{x} \text { entonces } I_{y}=I_{x}-t=\left\{r-t / r \in I_{x}\right\} \quad y \\
& \rho_{y}(s)=\rho_{x}(t+s) \forall s \in I_{y} \\
& \text { c) Diferenciabilidad respecto a las condiciones iniciales. } \\
& \text { El conjunto } D=\left\{(t, x) / x \in U, t \in I_{x}\right\} \text { es un abierto de } \mathbb{R}^{n+1} \quad y \\
& \text { la aplicación }
\end{aligned}
$$


$\rho: D C \mathbb{R}^{n+1} \rightarrow \mathbb{R}^{n},(t, x) \rightarrow \rho(t, x)=\rho_{x}(t)$ es de clase $c^{k}$ y satis face la ecuación

$$
D_{1} D_{2} \rho(t, x)=D X(\rho(t, x)) \cdot D_{2} \rho(t, x)
$$

donde $\mathrm{D}$ es la diferencial y $\mathrm{D}_{i}$ las derivadas parciales, considera das como matrices.

1.6. La aplicación $\rho$ se llama el flujo generado por $X$.

Observemos que $\rho$ satisface las propiedades de grupo a 1-parámetro es decir

$$
\left\{\begin{array}{l}
\rho(0, x)=x \\
\rho(t+S, x)=\rho(t, \rho(s, x))
\end{array}\right.
$$

$\operatorname{Si} I_{\mathbf{x}}=\mathbb{R} \quad \forall \mathrm{x}$.

En general se tiene $I_{x} \varsubsetneqq \mathbb{I R}$, por eso el flujo generado por $x$ se llama también flujo local o grupo local a 1-parámetro generado por $\mathrm{X}$.

Cuando $D=\mathbb{R} \times U$, tenemos un homomorfismo del grupo $(\mathbb{R},+)$ con el grupo $\operatorname{Dif}^{\mathrm{k}}(\mathrm{U})=\left\{\xi: U \rightarrow U / \xi\right.$ difeomorfismo de clase $\left.C^{\mathrm{k}}\right\}$ provisto de la operación composición

$$
\begin{aligned}
&(\mathbb{I R},+) \rightarrow \operatorname{Dif}^{k}(U) \\
& t \rightarrow \rho_{t}, \operatorname{con} \rho_{t}(x)=\rho(t, x) \\
& \text { observemos que } \rho_{t+s}=\rho_{t} \circ \rho_{s} y \rho_{t}^{-1}=\rho_{-t}
\end{aligned}
$$

La parte (a) del Teorema 1.5 son los clásicos Teoremas de Existencia y unicidad de ecuaciones diferenciales debidos a Cauchy, Peano, Picard, Lipschitz, Anzela, Weierstrass, Legendre, Lindelöff, etc. Un excelente tratamiento se encuentra en el libro de coddington y Levinson. En el capítulo 2 se da una hermosa demostración.

La parte (b) del Teorema 1.5 se desprende de lo siguiente. Consideremos $\left\{\begin{array}{l}x^{\prime}=x(x) \\ \rho_{(0)}=\rho_{x}(t)\end{array} \quad t\right.$, fijo 
obviamente $\rho_{x}(t+s)$ es solución, pues $\frac{d \rho_{x}}{d s}(t+s)=x\left(\rho_{x}(t+s)\right.$ $y \rho_{x}(t+0)=\rho_{x}(t)$.

Por otro lado $\rho_{y}(s)$ también es solución, pues $\frac{d \rho_{y}}{d s}(s)=x\left(\rho_{y}(s)\right.$ ) $y \rho_{y}(0)=y=\rho_{x}(t)$. Entonces por la parte $(a)$

$\rho_{x}(t+s)=\rho_{y}(s) \quad \forall s \in I_{x}-t=I_{y}$.

La parte (c), es mucho más delicada y la veremos en el capítulo 2.

1.7. Ejemplo. Consideremos la ecuación diferencial lineal $\mathbf{x}^{\prime}=\mathrm{Ax}$ donde $A$ matriz real de $\mathrm{n} \times \mathrm{n}$.

Sea $\phi(t)$ una matriz fundamental deं $\mathrm{x}^{\prime}=\mathrm{Ax} \operatorname{con} \phi(0)=I$ entonces $\phi(t)$ cumple

1. $\phi^{\prime}(t)=A \phi(t), \phi(0)=I$

2. $\forall t, s \in \mathbb{R}, \phi(t+s)=\phi(t) \phi(s)$

3. $[\phi(t)]^{-1}=\phi(-t)$

4. La serie $\sum_{m=0}^{\infty} \frac{t^{m} A^{m}}{m !}$ converge uniformemente sobre compactos a $\phi(t)$

$\phi(1):=$ exponencial de la matriz $A$, se denota por $e^{A}$

Entonces el flujo del campo $x^{\prime}=A x$ es $\rho: \mathbb{R}^{x} \mathbb{R}^{n} \rightarrow \mathbb{R}^{n}$

con $\rho(t, x)=e^{t A} x$, que se llama Flujo Lineal

1.8. Corolario. Sea $x$ un campo de clase $c^{k}, k \geqslant 1, U \subseteq \mathbb{R}^{n}$

Sea $x \in \Delta y I_{x}=\left(w_{-}(x), w_{+}(x)\right)$ y tal que $w_{+}(x)<\infty$

Entonces $\rho_{X}(t)$ tiende a $\partial U$ (la frontera de $U$ ) cuando $t$ tiende a $w_{+}(t)$. Es decir: $\forall \mathrm{K} \leqslant U$ compacto existe $\varepsilon>0$ tal que si

$t \in\left[w_{+}(x)-\varepsilon, w_{+}(x)\right)$, entonces

$$
\rho_{\mathrm{X}}(t) \notin \mathrm{K}
$$


Demostración: Si no. Supongamos exista $\mathrm{K} \subseteq$ U compacto y una sucesión $\left\{t_{n}\right\}_{n \in \mathbb{N}}, t_{n} \rightarrow w_{+}(x)<\infty$ tal que $\rho_{x}\left(t_{n}\right) \in k \forall n \in \mathbb{N}$. Luego existe $\left\{t_{n^{\prime}}\right\} \subseteq\left\{t_{n}\right\}$ subsucesión tal que $\lim _{n^{\prime} \rightarrow \infty} \rho_{x}\left(t_{n^{\prime}}\right)=x_{0} \in K$

Sean $b>0, \alpha>0$ tales que $B_{b} \times I_{\alpha} \subseteq D$ con

$\mathrm{B}_{\mathrm{b}}=\left\{\mathrm{y} \in \mathbb{R}^{\mathrm{n}} /|| \mathrm{y}-\mathrm{x}_{0}|| \leqslant \mathrm{b}\right\} \underline{\mathrm{c}} \mathrm{U} ; \mathrm{I}_{\alpha}=\{\mathrm{t} \in \mathbb{R} /|\mathrm{t}|<\alpha\}$

esto es posible pues $D$ es abierto.

Para $n_{0}$ suficientemente grande definamos $y:=\rho_{x}\left(t_{n_{0}}\right)$

Entonces para $0<s<\alpha$, se tiene $(y, s) \in B_{b} x I_{\alpha} C D$

luego podemos definir

$$
\begin{aligned}
\rho_{y}(s) & =\rho(s, y)=\rho\left(s, \rho_{x}\left(t_{n_{0}}\right)\right)=\rho\left(s, \rho\left(t_{n_{0}}, x\right)\right) \\
& =\rho\left(s+t_{n_{0}}, x\right)=\rho_{x}\left(s+t_{n_{0}}\right)
\end{aligned}
$$

Luego como $n_{0}$ es suficientemente grande $y t_{n} \rightarrow w_{+}(x)$ se tiene $s+t_{n_{0}}>w_{+}(x)$. Lo que contradice la maximalidad de $I_{x}$.

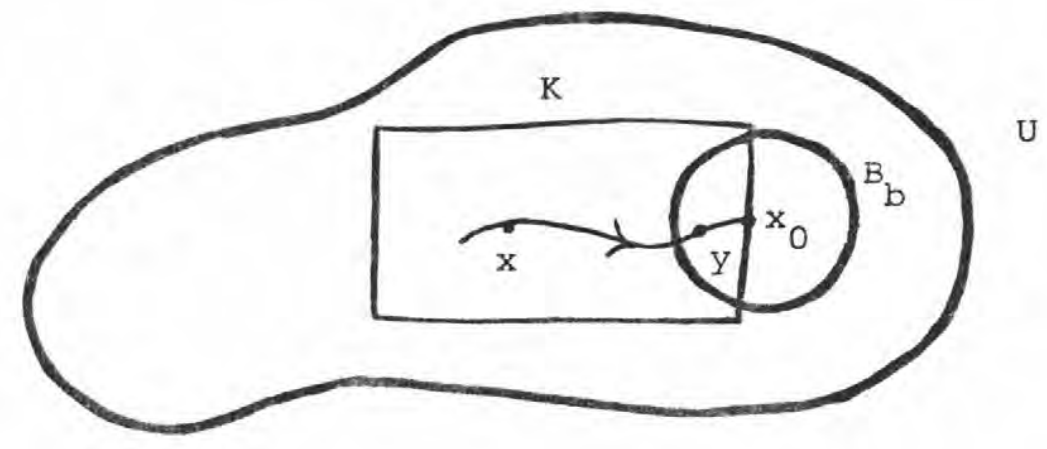

Lo mismo ocurre si $w_{-}(x)>-\infty$ y $t \rightarrow w_{-}(x)$ 
1.9. Corolario: Sea $U=\mathbb{R}^{\mathrm{n}}$ y $\|\mathrm{X}(\mathrm{x})\|<\mathrm{M} \quad \forall \mathrm{x} \in \mathbb{R}^{\mathrm{n}}$.

Entonces $I_{x}=\mathbb{R} \forall x \in \mathbb{R}^{n}$.

Demostración: Supongamos $w_{+}(x)<\infty$ para algún $x \in \mathbb{R}^{n}$.

Sabemos $\rho_{t}(x)=x+\int_{0}^{t} x\left(\rho_{t}(s)\right) d s$, tenemos

$\left\|\mathrm{x}-\rho_{t}(s)\right\|=|| \int_{0}^{t} \mathrm{x}\left(\rho_{t}(s)\right) d s \| \leqslant M t \leqslant M w_{+}(x)$

es decir $\forall t \in\left[0, w_{+}(x)\right), \rho_{t}(x) \in B\left(x, M w_{+}(x)\right)$, la bola con centro en $\mathrm{x} y$ radio $\mathrm{M} \mathrm{w}_{+}(\mathrm{x})$, por 10 tanto $\rho_{t}(\mathrm{x})$ no va a la frontera de $\partial \mathbb{R}^{n}$ cuando $t \rightarrow w_{+}(x)$ lo que contradice 1.7 . Por lo tanto $w_{+}(x)=+\infty$. Analogamente $w_{-}(x)=-\infty \forall x \in \mathbb{R}^{n}$.

1.10. Corolario: Si $p$ es una solución de $x^{\prime}=x(x)$ definida en su intervalo máximo $I, y$ existen $t_{1}, t_{2} \in I, t_{1} \neq t_{2}$ con

$\rho\left(t_{1}\right)=\rho\left(t_{2}\right)$. Entonces $I=\mathbb{R} y \rho(t+c)=\rho(t) \quad \forall t \in \mathbb{R}$ con $c=t_{2}-t_{1}$. Es decir $\rho$ es periódica.

Demostración: $\quad$ sea $\psi:\left[t_{2}, t_{2}+c\right] \rightarrow \mathbb{R}^{n}$

$$
t \rightarrow \psi(t)=\rho(t-c)
$$

se tiene $\psi^{\prime}(t)=\rho^{\prime}(t-c)=x(\rho(t-c))=x(\psi(t))$

luego $\psi$ es solución de $x^{\prime}=x(x)$ y $\psi\left(t_{2}\right)=\rho\left(t_{2}-c\right)$

$=\rho\left(t_{2}-\left(t_{2}-t_{1}\right)\right)=\rho\left(t_{1}\right)=\rho\left(t_{2}\right)$. Entonces por unicidad de solucio nes se tiene $\left[t_{2}, t_{2}+c\right] \underline{c} I y \quad \rho(t)=\psi(t+c)=\rho(t+c)$

si $t \in\left[t_{1}, t_{2}\right]$. Prosiguiendo de esta manera se tiene $I=R \quad y$ $\rho(t+c)=\rho(t) \quad \forall \quad t \in \mathbb{R}$. 


\section{§. 2. DIFERENCIABIIIDAD DE LOS FLUJOS GENERADOS POR CAMPOS VECTOFIALES.}

El objetivo de este capítulo es demostrar la parte (c) del Teorema 1.5. El tratamiento es totalmente autosuficiente e incluirá (a) $y$ (b).

2.1. Teorema de Contracción en la fibras.

Sean $(x, d),(\dot{x}, d)$ espacios métricos completos $y$

$\hat{\mathrm{F}}: \mathrm{X} \mathrm{x} \hat{\mathrm{X}} \rightarrow \mathrm{X} \mathrm{x} \dot{\mathrm{X}}$

$(x, \dot{x}) \rightarrow(F(x), F(x, \dot{x}))$ Supongamos que

a) $F: X \rightarrow X$ tiene un punto fijo atractor, es decir existe $p \in X$ con $F(p)=p y \lim _{n \rightarrow \infty} F^{n}(x)=p \quad \forall x \in X$

b) La aplicación $x \rightarrow \dot{F}(x, \dot{x})$ continua en $x \quad \forall \dot{x} \in \dot{x}$

c) $\forall x \in X, l a$ aplicación $\dot{F}_{x}: \dot{x} \rightarrow \dot{x}$ definida por

$\dot{\mathrm{F}}_{\mathrm{x}}(\dot{\mathrm{x}})=\dot{\mathrm{F}}(\mathrm{x}, \dot{\mathrm{x}})$ es una $\lambda$-contracción con $\lambda<1$, es decir

$d\left(\dot{F}_{x}(\dot{x}), \dot{F}_{x}(\dot{y})\right) \leqslant \lambda d(\dot{x}, \dot{y}) \quad \forall \dot{x}, \dot{y} \in \dot{x}$

Entonces:

Si $\dot{p}$ denota el único punto fijo atractor de $\dot{F}_{p}$ (que existe por ser $\lambda$-contracción) se tiene que el punto $\hat{p}=(p, \dot{p})$ es un punto fijo atractor de $\hat{F}$.

Demostración:

La demostración está basada en los 2 lemas siguientes

Lema 1 ; Sea $\left\{C_{n}\right\}_{n \geqslant 0}$ sucesión de numeros reales no negativos tales que $c_{n} \rightarrow 0 . \quad Y$ sea $\lambda$ tal que $0<\lambda<1$.

Entonces la sucesión

$$
\sigma_{n}=\sum_{i-0}^{n} \lambda^{n-i} c_{i} \quad \rightarrow_{n \rightarrow \infty} 0
$$

Prueba: Interesante ejercicio de cálculo 
Lema 2: Sea $(Y, d)$ espacio métrico completo.

$\left\{F_{n}\right\}$ sucesión de $\lambda$-contracciones.

i) Si $\forall y \in Y, F_{n}(y) \rightarrow F_{w}(y)$. Entonces $F_{w}$ también es una $\lambda$-contracción.

ii) Si $y_{w}$ es el único punto fijo atractor de $F_{W}$. Entonces

$\forall y_{0} \in Y, l a$ sucesión $\left\{y_{n}\right\}$ definida por

$$
\begin{aligned}
& y_{0}=y_{0} \\
& y_{1}=F_{1}\left(y_{0}\right) \\
& y_{2}=F_{2}\left(y_{1}\right) \\
& \vdots \\
& y_{n}=F_{n}\left(y_{n-1}\right)
\end{aligned}
$$

converge para $\mathrm{y}_{\mathrm{w}}$ cuando $\mathrm{n} \rightarrow \infty$.

\section{Prueba:}

i) $d\left(F_{w}(y), F_{w}\left(y^{\prime}\right) \leqslant d\left(F_{w}(y), F_{n}(y)\right)+d\left(F_{n}(y), F_{n}\left(y^{\prime}\right)\right)\right.$

$$
+d\left(F_{n}\left(y^{\prime}\right), F_{w}\left(y^{\prime}\right)\right)
$$

$\leqslant \varepsilon / 2+\lambda d\left(y, y^{\prime}\right)+\varepsilon / 2$ si n suficientemente grande

$\therefore \quad d\left(F_{w}(y), F_{w}\left(y^{\prime}\right)\right) \leqslant \lambda d\left(y, y^{\prime}\right)$ ed uná $\lambda$-contracción

ii) $y_{n}=F_{n} \circ F_{n-1} \circ \ldots \circ F_{1}\left(y_{0}\right) y$ consideremos

$d\left(y_{n}, y_{w}\right) \leqslant d\left(F_{n} \circ \ldots \circ F_{1}\left(y_{0}\right), F_{n} \circ \ldots \circ F_{1}\left(y_{w}\right)\right)+d\left(F_{n} \circ \ldots \circ F_{1}\left(y_{w}\right), y_{w}\right)$

$\leqslant \lambda d\left(F_{n-1} \circ \ldots_{1} \circ F_{0}\left(y_{n-1}\right), F_{n o F}\left(y_{w}\right)\right)+d\left(F_{n} \circ \ldots \circ F_{1}\left(y_{w}\right), F_{n}\left(y_{w}\right)\right)+d\left(F_{n}\left(y_{w}\right), y_{w}\right)$

$\leqslant \lambda^{n} d\left(y_{0}, y_{w}\right)+\lambda d\left(F_{n-1} 0 \ldots o F_{1}\left(y_{w}\right), y_{w}\right)+d\left(F_{n}\left(y_{w}\right), y_{w}\right)$

$\leqslant \lambda^{n} d\left(y_{0}, y_{w}\right)+d\left(F_{n}\left(y_{w}\right), y_{w}\right)+\lambda\left(F_{n-1} 0 \ldots o F_{1}\left(y_{w}\right), F_{n-1}\left(y_{w}\right)\right)+$

$$
\left.+d\left(F_{n-1}\left(y_{w}\right), y_{w}\right)\right]
$$




$$
\begin{aligned}
\leqslant \lambda^{n} d\left(y_{0}, y_{w}\right)+d\left(F_{n}\left(y_{n}\right), y_{w}\right) & +\lambda d\left(F_{n-1}\left(y_{w}\right), y_{w}\right)+ \\
& +\lambda^{2} d\left(F_{n-2} \circ \ldots \circ F_{1}\left(y_{w}\right), F_{n-1}\left(y_{w}\right)\right.
\end{aligned}
$$

$\leqslant \lambda^{n} d\left(y_{0}, y_{w}\right)+\sum_{i=0}^{n-1} \lambda^{i} d\left(F_{n-i}\left(y_{w}\right), y_{w}\right)$

$\lambda^{\mathrm{n}} \mathrm{d}\left(\mathrm{y}_{0}, \mathrm{y}_{\mathrm{w}}\right) \rightarrow 0 \quad$ pues $\lambda<1$

$\sum_{i=0}^{n-1} \lambda^{i} d\left(F_{n-i}\left(y_{w}\right), y_{w}\right) \rightarrow 0$ por Lema 1 con $c_{n}=d\left(F_{n}\left(y_{w}\right), y_{w}\right)$

$y C_{n} \rightarrow 0$ pues $F_{n}\left(y_{w}\right) \rightarrow F_{w}\left(y_{w}\right)=y_{w}$

Por 10 tanto $\mathrm{y}_{\mathrm{n}} \rightarrow \mathrm{y}_{\mathrm{w}}$.

\section{Demostremos ahora el Teorema:}

$$
\text { Sea } \begin{aligned}
\hat{x}_{0}=\left(x_{0}, \dot{x}_{0}\right), x_{n}=F^{n}\left(x_{0}\right) \\
\hat{F}^{1}\left(\hat{x}_{0}\right)=\left(F\left(x_{0}\right), \dot{F}_{x_{0}}\left(\dot{x}_{0}\right)\right)=\left(x_{1}, \dot{F}_{x_{0}}\left(\dot{x}_{0}\right)\right) \\
\hat{F}^{2}\left(\hat{x}_{0}\right)=\hat{F}\left(x_{1}, \dot{F}_{x_{0}}\left(\dot{x}_{0}\right)\right)=\left(F\left(x_{1}\right), \dot{F}_{x_{1}}\left(\dot{F}_{x_{0}}\left(\dot{x}_{0}\right)\right)\right) \\
\quad=\left(x_{2}, \dot{F}_{x_{1}} \circ \dot{F}_{x_{0}}\left(\dot{x}_{0}\right)\right. \\
\vdots \\
\hat{F}_{n}\left(\hat{x}_{0}\right)=\left(x_{n}, \dot{F}_{x_{n-1}} \circ \dot{F}_{x_{n-2}} \circ \ldots \circ \dot{F}_{x_{1}} \circ \dot{F}_{x_{0}}\left(\dot{x}_{0}\right)\right)
\end{aligned}
$$

En la notación del Lema 2, tomemos

$$
\mathrm{y}_{\mathrm{n}}=\dot{\mathrm{F}}_{\mathrm{x}_{\mathrm{n}-1}} \circ \cdots \circ \dot{\mathrm{F}}_{\mathrm{x}_{0}}\left(\dot{\mathrm{x}}_{0}\right) \rightarrow \dot{\mathrm{F}}_{\mathrm{p}}(\dot{\mathrm{p}})=\dot{\mathrm{p}}
$$

Entonces $\hat{\mathrm{F}}^{\mathrm{n}}\left(\hat{\mathrm{x}}_{\mathrm{o}}\right)=\left(\mathrm{x}_{\mathrm{n}}, \mathrm{y}_{\mathrm{n}}\right) \rightarrow(\mathrm{p}, \dot{\mathrm{p}})$. 


\subsection{Teorema local de difernciabilidad.}

Sea $f: U \subseteq \mathbb{R}^{n} \rightarrow \mathbb{R}^{n}$ de clase $C^{1}$. Entonces $\forall x_{0} \in U$ existen $\alpha, \beta \in \mathbb{R}^{+}$con $I_{\alpha} \times B_{\beta}=\left\{(t, x) /|t|<\alpha,\left\|x-x_{0}\right\|<\beta\right\} \underline{c} U$ y una única aplicación $\rho: I_{\alpha} \times B_{\beta} \rightarrow U$ de clase $C^{1}$ tal que

1) $\left.D_{1} \rho(t, x)=\frac{\partial \rho}{\partial t}(t, x)=f(\rho / t, x)\right)$ y $\rho(0, x)=x$

2) $D_{1} D_{2} \rho(t, x)=D f(\rho(t, x)) \cdot D_{2} \rho(t, x)$

$\forall(t, x) \in I_{\alpha} \times B_{\beta}$

Demostración:

Sea $b>0$ tal que $\bar{B}_{b}=\left\{x /|| x-x_{0}|| \leqslant b\right\} \underline{c} U$

$\operatorname{sean} m=\sup || f(x)||, x \in \bar{B}_{b}$

$I=\sup || D f(x)||, x \in \bar{B}_{b}$

$\operatorname{sean} \alpha, \beta$ tales que $\alpha m+\beta<b, \lambda=1 \alpha<1$

$x=\left\{\rho: I_{\alpha} \times B_{B} \rightarrow \bar{B}_{b} / \rho\right.$ continua $\}$. En $x$ tenemos la siguiente métrica

$d(\rho, \psi)=\sup || \rho(t, x)-\psi(t, x) \|(t, x) \in I_{\alpha} \times B_{B}$

$L=\left\{L: \mathbb{R}^{\mathrm{n}} \rightarrow \mathbb{R}^{\mathrm{n}} / \mathrm{L}\right.$ lineal $\},\|I\|\left\|_{L}=\operatorname{Sup}_{\| \mathrm{x} \mid}|| \mathrm{I}(\mathrm{x})\right\|$

Sea $\dot{x}=\left\{\dot{\rho}: I_{\alpha} \times B_{\beta} \rightarrow L / \dot{\rho}\right.$ continua $y$ acotada $\}$

En $\dot{x}$ tenemos la métrica

$d(\dot{\rho}, \dot{\psi})=\sup _{(t, x) \in I_{\alpha} x_{B}}\|\dot{\rho}(t, x)-\psi(t, x)\|_{L}$

Definamos $F: x \rightarrow x \operatorname{como} F(\rho)(t)=x+\int_{0}^{t} f(\rho(s, x)) d s$

y $\dot{F}: x x \dot{x} \rightarrow \dot{x} \operatorname{como} \hat{F}(\rho, \dot{p})(t, x)=I+\int_{0}^{t} D f(\rho(s, x)) \cdot \dot{\rho}(s, x) d s$

I identidad de $L_{i}$

Demostremos que $\hat{F}:=(F, F)$ satisface las hipótesis de 2.1. 
i) $F$ es una $\lambda$-contracción:

$d(F(p), F(\psi))=\sup \left\|\int_{0}^{t}[f(\rho(s, x))-f(\psi(s, x))] d s\right\|$

$\underset{\sup \mid}{\text { T.V.Medio }} \int_{0}^{t} \ell|| \rho(\mathrm{x})-,\psi(\mathrm{s}, \mathrm{x})|| \mathrm{ds} \mid \leqslant \alpha \ell \mathrm{d}(\alpha, \psi)$

$=\lambda d(\alpha, \psi) \quad \therefore \quad F$ tiene un único punto fijo atractor $\rho \in \mathrm{X}$

ii) $\rho \rightarrow \dot{F}(\rho, \ddot{\rho})$ es continua en $x \quad \forall \dot{\rho}$ e $\dot{x}$, pues

Df es uniformemente continua en $\bar{B}_{b}$

iii) d $\left(\dot{F}_{\rho}(\dot{\rho}), \dot{\mathrm{F}}_{\rho}(\dot{\psi})\right)=$

$=\left.\sup || \int_{0}^{t} \operatorname{Df}(\rho(s, x))[\dot{\rho}(s, x)-\psi(s, x)] d s\right|_{L} \leqslant \lambda d(\dot{\rho}, \dot{\psi})$

El punto fijo atractor de $\hat{F}$ es de la forma $\hat{\rho}=(\rho, \dot{\rho})$

donde $F(\rho)=\rho$ es decir:

$F(\rho)(t, x)=x+\int_{0}^{t} f(\rho(s, x)) d s=\rho(t, x)$

Luego $\rho(0, x)=x y$ derivando respecto de $t(*)$ tenemos

$D_{1} \rho(t, x)=f(\rho(t, x))$ es decir hemos demostrado (1) pues $\rho$ es unica por ser único el punto fijo de $F y$ es continua en $I_{\alpha} \times B_{\beta}$ por ser elemento de $x$.

Demostremos que $\rho$ es de clase $\mathrm{C}^{1}$ con respecto a $\mathrm{x} y$ que

$\mathrm{D}_{2} \rho=\dot{\rho}$

Claramente $\rho$ es de clase $C^{1}$ respecto a $t$, por $\operatorname{ser} D, \rho=f \circ \rho$ que es continua.

$$
\text { Sea } \hat{\rho}_{n}=\left(\rho_{n}, \dot{\rho}_{n}\right)=\hat{F}^{n}\left(\hat{\rho}_{0}\right) \text { donde } \rho_{0}(t, x)=x
$$

y $\dot{\rho}_{0}(t, x)=I$. Por 10 construido anteriormente $((\rho, \dot{\rho})$ punto fijo atrac tor de $\hat{F})$, tenemos $\rho_{n} \rightarrow \rho, \dot{\rho}_{n} \rightarrow \dot{\rho}$ uniformemente en $I_{\alpha} \times B_{B}$. 
Aun más, $\rho_{n}$ es de clase $C^{1}$ y $D_{2} \rho_{n}=\dot{\rho}_{n}$, esto se puede verificar trivialmente por inducción

$$
\begin{aligned}
& \mathrm{n}=0 ; \rho_{0}(t, x)=x \text { clase } c^{1} \text { y } D_{2} \rho_{0}(t, x)=I=\dot{\rho}_{0}(t, x) \\
& \mathrm{n}=1 ; \hat{\mathrm{F}}\left(\hat{\rho}_{0}\right)=\mathrm{F}\left(\rho_{0}, \dot{\rho}_{0}\right)=\left(\mathrm{F}\left(\rho_{0}\right), \dot{\mathrm{F}}\left(\rho_{0}, \dot{\rho}_{0}\right)\right) \\
& =\left(x+\int_{0}^{t} f\left(\rho_{0}(s, x)\right) d s, I+\int_{0}^{t} D f\left(\rho_{0}(s, x)\right) \cdot \dot{\rho}_{0}(s, x) d s\right) \\
& =(x+f(x) t, I+D f(x) t):=\left(\rho_{1}, \dot{\rho}_{1}\right)
\end{aligned}
$$

Luego $\forall n, D_{2} \rho_{n}=\dot{\rho}_{n} \in \dot{x}$, por lo tanto $D_{2} \rho_{n}$ continua.

$y \lim D_{2} \rho_{n}=D_{2} \lim \rho_{n}=D_{2} \rho$

$\mathrm{y} \lim \dot{\rho}_{\mathrm{n}}=\dot{\rho}$ (se puede hacer por continuidad uniforme)

Entonces $D_{2} \rho=\dot{\rho} e \dot{x}$, por 10 tanto continua en $I_{\alpha} \times B_{B}$

Para demostrax la fórmula (2), derivemos respecto de $t$ la relación:

$$
\begin{aligned}
D_{2} \rho(t, x) & =\dot{\rho}(t, x)=\dot{F}(\rho, \dot{\rho})(t, x)=\dot{F}\left(\rho, D_{2} \rho\right)(t, x) \\
& =I+\int_{0}^{t} \operatorname{Df}(\rho(s, x)) D_{2} \rho(s, x) d s
\end{aligned}
$$

Luego $D_{1} D_{2} \rho(t, x)=D f(\rho(t, x)) D_{2} \rho(t, x)$

$\forall(t, x) \in I_{\alpha} x B_{\beta}$. 


\subsection{Teorema Global de diferenciabilidad (Teorema 1.5)}

$f: U \subseteq \mathbb{R}^{n} \rightarrow \mathbb{R}^{n}, f$ de clase $c^{k}, k \geqslant 1$ en el abierto $U$. Entonces

a) $\forall x \in U$, existe $I_{x} \subseteq \mathbb{R}$ intervalo abierto, existe

$\rho_{\mathrm{X}}: I_{\mathrm{X}} \rightarrow \mathrm{U}$ curva integral máxima del campo $\mathrm{f}$ pasando por $x$.

Es decir $\rho_{x}$ satisface la ecuación $\dot{x}=f(x), x(0)=x$

b) $\operatorname{Si} y=\rho_{x}(t), t \in I_{x}$, entonces

$$
I_{y}=I_{x}-t=\left\{\tau-t / \tau \in I_{x}\right\} y \quad \rho_{y}(s)=\rho_{x}(t+s) \forall s \in I y
$$

c) El conjunto $\mathrm{D}\left\{(t, \mathrm{x}) / \mathrm{x} \in \mathrm{U}, t \in \mathrm{I}_{\mathrm{x}}\right\}$ es un abierto de $\mathbb{R}^{\mathrm{n}+1}$ y la aplicación $\rho: D \rightarrow \mathbb{R}^{n}$ definida por

$\rho(t, x)=\rho_{X}(t)$ es de clase $c^{k}$.

La demostración del teorema se desprende de las siguientes dos proposiciones.

Proposición 1.

Sea $S_{x}=\left\{\psi: I_{\psi} \rightarrow U / \psi\right.$ solución del problema $\left.x^{\prime}=f(x), x(0)=x\right\}$, $x \in U$. Definamos $I_{x}=\underset{\psi \in S_{x}}{U} I_{\psi}$

\section{Entonces:}

(i) $\rho_{x}: I_{x} \rightarrow U$ definida por $\rho_{x}(t)=\psi(t)$ si $t \in I_{\psi}$ es la única curva integral máxima def. por $\mathrm{x}$.

(ii) Si $t \in I_{x}, y=\rho_{x}(t)$, entonces $I_{y}=I_{x}-t=\left\{s-t / s \in I_{x}\right\}$ $y \forall s \in I_{y}$ se tiene $\rho_{x}(t+s)=\rho_{y}(s)$ 


\section{Prueba:}

(i) Es suficiente verificar que $\rho_{x}$ está bien definida. Es decir si $\psi_{1}, \psi_{2} \in S_{x}$ entonces $\psi_{1}=\psi_{2}$ en $(a, b)=I_{\psi_{1}} \cap I_{\psi_{2}}$ pues $\mathrm{si}$

$A=\left\{t \in(a, b) / \psi_{1}(t)=\psi_{2}(t)\right\}$ es un conjunto cerrado $y$ no vacío. Pro bemos que $A$ es abierto. Sea $t^{\prime}$ e A Y $y=\psi_{1}\left(t^{\prime}\right)=\psi_{2}\left(t^{\prime}\right)$. Entonces por el Teorema local de diferenciabilidad (2.2) existe una única curva integral $\psi$ de $x^{\prime}=f(x), x(0)=y$ definida en un intervalo abierto I $\subseteq \mathbb{R}$.

$$
\begin{aligned}
& \text { Notemos que } \tilde{\psi},(s)=\psi_{1}\left(t^{\prime}+s\right) \text {, entonces } \tilde{\psi}_{1} \in s \text { y pues } \\
& \frac{d}{d s} \tilde{\psi},(s)=\frac{d}{d s} \psi_{1}\left(t^{\prime}+s\right)=f\left(\psi_{1}\left(t^{\prime}+s\right)\right)=f(\tilde{\psi},(s)) \text { y por unicidad se } \\
& \text { tiene } \psi_{1}=\psi \text { en }(a, b) \cap\left(I+t^{\prime}\right) .
\end{aligned}
$$$$
\text { Analogamente } \tilde{\psi}_{2}(s)=\psi_{2}\left(t^{\prime}+s\right), \tilde{\psi}_{2} \in s_{y} y \psi_{2}=\psi \text { en }(a, b) \cap\left(I+t^{\prime}\right) \text {. }
$$$$
\text { Luego } \psi_{1}=\psi_{2} \text { en }(a, b) \Omega\left(I+t^{\prime}\right) \text { es decir A es abierto. Por conexidad }
$$$$
A=(a, b) \text {. }
$$

(ii) Tenemos $\rho_{y}(s)=\rho_{x}(t+s)$ Iuego $\rho_{y}(s)$ está definida para $s I_{x}{ }^{-t}$, donde $I_{x}-t \subseteq I_{y}$. Por otro lado $\psi_{y}(-t)=x y \rho_{x}(s)=\rho_{y}(-t+s)$ donde $\rho_{x}(s)$ está definida $\forall s \in I_{y}+t$. Luego $I_{y}+t c I_{x}$ es $\operatorname{decir} I_{y} \subseteq I_{x}-t$. Entonces $I_{y}=Y_{x}-t$.

Esta proposición es válida para f de clase $\mathrm{C}^{1}$

Proposición 2.

Sea $f: U \subseteq \mathbb{R}^{n} \rightarrow \mathbb{R}^{n}$ de clase $C^{1}$ en el abierto $U$.

Entonces $D=\left\{(t, x) / x \in U, t \in I_{x}\right\}$ es un abiexto de $\mathbb{R}^{n+1}$ y el flujo $\rho(t, x)=\rho_{x}(t)$ es de clase $C^{1}$ en $D y$ satisface 


$$
D_{1} D_{2} \rho(t, x)=D f(\rho(t, x)) D_{2} \rho(t, x) \ldots
$$

$\forall(t, x)$ e $D$.

( $I_{x}$ es el intervalo de la solución $\rho_{x}$ máxima de $S_{x}$ )

\section{Prueba:}

$\mathrm{C}^{+}=\left\{t \in I_{x_{0}} / t>0 y\right.$ existe un abierto $B_{t} c \mathbb{R}^{n}, x_{0} \in B_{t}$ tal que

$$
\left.[0, t] \times B_{t} \subseteq D y \rho \text { es de clase } C^{1} \text { y satisface }(*) \text { en }(0, t) \times B_{t}\right\}
$$

Por el teorema local de diferenciabilidad (2.2), $\mathrm{c}^{+} \neq \phi$.

Sea $\mathrm{S}=$ supremo de $\mathrm{C}^{+}$. Demostremos que $\mathrm{s}$ es el extremo superior de $I_{\mathrm{x}}$. Si no lo fuera, $s \in I_{x}$, sea $x_{1}=\rho\left(S, x_{0}\right)$.

Por el teorema local de diferenciabilidad (2.2) existe I x B vecindad de $\left(0, x_{1}\right)$ en la cual $p$ satisface $\left(^{*}\right)$. Sea d el largo de $I y$ $\mathrm{U} \in \mathbb{R}$ tal que $\mathrm{u}<\mathrm{s}$ y $\mathrm{s}-\mathrm{u}<\mathrm{d} / 2$ y $\underset{\mathrm{B}}{\sim}$ una vecindad de $\mathrm{x}_{0}$ tal que $\rho(u, y) \in B \quad \forall y \in \stackrel{\sim}{B}$.

Si $y \in \widetilde{B}$ y $t \in[0, u+d / 2]$ tenemos por la proposición 1 que $\rho(t, y)=\rho(t-u, \rho(u, y)) \therefore \rho$ es de clase $c^{1}$ en $(0, u+d / 2) \times \tilde{B}$. Vea mos que $\rho$ satisface $(*)$ en este conjunto como $\rho(t, x)=\rho(t-u, \rho i u, x))$ derivando respecto a $\mathrm{x}$

$$
D_{2} \rho(t, x)=\left[D_{2} \rho(t-u, \rho(u, x))\right] D_{2} \rho(u, x) \ldots(* *)
$$

derivando ahora respecto a $t y$ observando que $t-u \in C^{+}$

$$
\begin{aligned}
D_{1} D_{2} \rho(t, x) & =\left[D_{1} D_{2} \rho(t-u, \rho(u, x))\right] D_{2} \rho(u, x)+0 \\
& =\left[D f(\rho(t, x)) D_{2} \rho(t-u, \rho(u, x))\right] D_{2} \rho(u, x) \\
& =D f(\rho(t, x)) D_{2} \rho(t, x) \quad \text { (por **) }
\end{aligned}
$$

Luego $\mathrm{u}+\mathrm{d} / 2 \mathrm{e}^{+} \mathrm{y}$ es mayor que $\mathrm{s}$. Contradicción.

Analogamente se define $\mathrm{C}^{-} \mathrm{y}$ se tiene $\mathrm{I}_{\mathrm{x}_{0}}=\mathrm{C}^{-} \mathrm{U} \mathrm{C}^{+}$ 
Demostración de 2.3. Inducción sobre k.

$\mathrm{k}=1$. La proposición 2 .

$\mathrm{k}>1$, supongámoslo válido para $\mathrm{k}-1$. Consideremos el campo

$F=(f, D f)$ de clase $c^{k-1}$ en $U \times \mathbb{R}^{n^{2}}$ definido por

$F(x, L)=(f(x), D f(x) \cdot L), L \in L$.

Por la proposición 2 y la hipótesis de inducción aplicada a F tenemos que el flujo

$\phi(t, y, Y)=\left(\rho(t, y), D_{2} \rho(t, y) \cdot Y\right)$ es de clase $c^{k-1}$ en $D^{\prime}=D x \mathbb{R}^{n^{2}}$

Luego $D_{2} \rho$ es de clase $C^{k-1}$ en $D$, también $D_{1} \rho=f \circ \rho$ es de clase $C^{k-1}$ pues $f$ de clase $C^{k}$ y $\rho$ de clase $c^{k-1}$

Luego $\rho$ de clase $C^{k}$ en $D$.

\section{§.3. RETRATO DE FASE DE UN CAMPO VECTORIAL.}

3.1. La imagen de una curva integral de $x$ por el punto $p \in U$ se llama órbita de $\mathrm{x}$ por el punto $\mathrm{p}, \mathrm{y}$ se denota $\gamma_{\mathrm{p}}$. Es decir $\gamma_{p}=\left\{\rho(t, p) / t \in I_{p}\right\}$

3.2. Lema $q \in \gamma_{p} \Longleftrightarrow \gamma_{p}=\gamma_{q}$

Prueba: $\quad$ Si $q \in \gamma_{p}, q=\rho\left(t_{1}, p\right) y \rho(t, q)=\rho\left(t+t_{1}, p\right) y$

$I_{p}-t_{I}=I_{q}$

Luego dos órbitas de $\mathrm{x}$ coinciden o son disjuntas.

Entonces podemos descomponer $U$ en una unión disjunta de curvas diferenciales pudiendo ser cada una

a) Imagen biunívoca de un intervalo de $\mathbb{R}$,

b) Un punto, o

c) Difeomorfa a un círculo, correspondiendo a las alternativas del teorema 3.3 que sigue. 
En el caso b) $p=\gamma_{p}$ y la órbita se llama un punto singular.

En el caso c) la órbita se llama cerrada o periódica.

\section{3. Teorema:}

Si $\rho$ es una solución máxima de $x^{\prime}=x(x)$, entonces se verifica una unica de las siguientes alternativas.

a) $\rho$ es inyectiva.

b) $I=\mathbb{R}$ y $\rho$ es constante.

c) $I=\mathbb{R}$ y $\rho$ es periódica. Es decir existe $\tau>0$ tal que $\rho(t+\tau)=\rho(t)$ $\forall t \in \mathbb{R} \quad y \quad \rho\left(t_{1}\right) \neq \rho\left(t_{2}\right)$ si $\left|t_{1}-t_{2}\right|<\tau$

Demostración: Si $\rho$ no es inyectiva, $\rho\left(t_{1}\right)=\rho\left(t_{2}\right)$ para algún $t_{1} \neq t_{2}$ ' entonces por Corolario $1.10 \mathrm{I}=\mathbb{R}$ y $\rho(t+c)=\rho(t)$

$\forall t \in \mathbb{R}$ y $C=t_{2}-t_{1} \neq 0$

Sea $C=\{c \in \mathbb{R} / \rho(t+c)=\rho(t) \quad \forall t \in \mathbb{R}\}$. Demostremos que $C$ es un subgrupo aditivo cerrado de $\mathbb{R}$, luego (Teoría de Grupos!) C es de la forma $\tau \mathbb{z} \sigma \quad C$ es denso en $\mathbb{R}$ y por cerrado $C=\mathbb{R}$, lo que corres ponde a las alternativas b) y c).

Efectivamente $C$ es un subgrupo de $(\mathbb{R},+)$, pues si c, d $\in \mathrm{C}$ entonces $c+d \in C$ pues $\rho(t+c+d)=\rho(t+c)=\rho(t) \forall t \in \mathbb{R}$

$y-c \in C$ pues $\rho(t-c)=\rho(t-c+c)=\rho(t) \quad \forall t \in \mathbb{R}$

que $c \in c$.

Veamos que $C$ es cerrado. Si $c_{n} \in C, \underset{n \rightarrow \infty}{\lim } C_{n}=C$, demostramos

$$
\begin{aligned}
\rho(t+c) & =\rho\left(t+\lim _{n \rightarrow \infty} c_{n}\right)=\rho\left(\lim _{n \rightarrow \infty}\left(t+c_{n}\right)\right) \\
& =\lim _{n \rightarrow \infty} \rho\left(t+c_{n}\right)=\lim _{n \rightarrow \infty} \rho(t)=\rho(t) .
\end{aligned}
$$

3.4. Un conjunto abierto $U$, provisto de descomposición en órbitas de $X$ se llama retrato de fase de $\mathrm{x}$. 
Las órbitas son orientadas en el sentido de las curvas integrales del campo $\mathrm{x}$.

\subsection{Ejemplos:}

1.- Campos en la recta real. Sea $x: \mathbb{R} \rightarrow \mathbb{R}$ campo de clase $c^{k}$ supongamos $x^{-1}(0)=\left\{a_{1}<a_{2}<\ldots<a_{n}\right\}$ puntos singulares. Haga$\operatorname{mos} a_{0}=-\infty, a_{n+1}=\infty$

En cada intervalo $\left(a_{i}, a_{i+1}\right) i=0,1, \ldots, n$ el signo de $x$ es constante. Fijemos un intervalo $\left(a_{i}, a_{i+1}\right)$ donde $x$ es positivo. Entonces $\forall x \in\left(a_{i}, a_{i+1}\right)$ tenemos $\rho(t, x)$ estrictamente creciente en su intervalo máximo $I_{x}=\left(w_{-}(x), w_{+}(x)\right)$

Además afirmamos:

a) $\rho(t, x) \rightarrow a_{i}$ cuando $t \rightarrow w_{-}(x) ; \rho(t, x) \rightarrow a_{i+1}$ cuando $t \rightarrow w_{+}(x)$.

En efecto si $\rho(t, x) \rightarrow b>a_{i}$ cuando $t \rightarrow w_{-}(x)$ como $\rho(t, b)$ es estrictamente creciente se tiene $\gamma_{\mathrm{x}} \cap \gamma_{\mathrm{b}} \neq \phi$ es $\operatorname{decir} \gamma_{\mathrm{x}}=\gamma_{\mathrm{b}}$. Contradicción. Analogamente cuando $t \rightarrow w_{+}(x)$.

b) Si $i \geqslant 1$ tenemos $w_{-}(x)=-\infty$

Pues $\forall t \in I_{x}$, se tiene $\rho(t, x)>a_{i}>-\infty$ esto implica por la proposición 1 del Teorema 2.3 que $w_{-}(x)=-\infty$.

c) Si $i<n$, tenemos $w_{+}(x)=\infty$

Si $x$ es negativa en $\left(a_{i}, a_{i+1}\right)$ los resultados son recíprocos. 

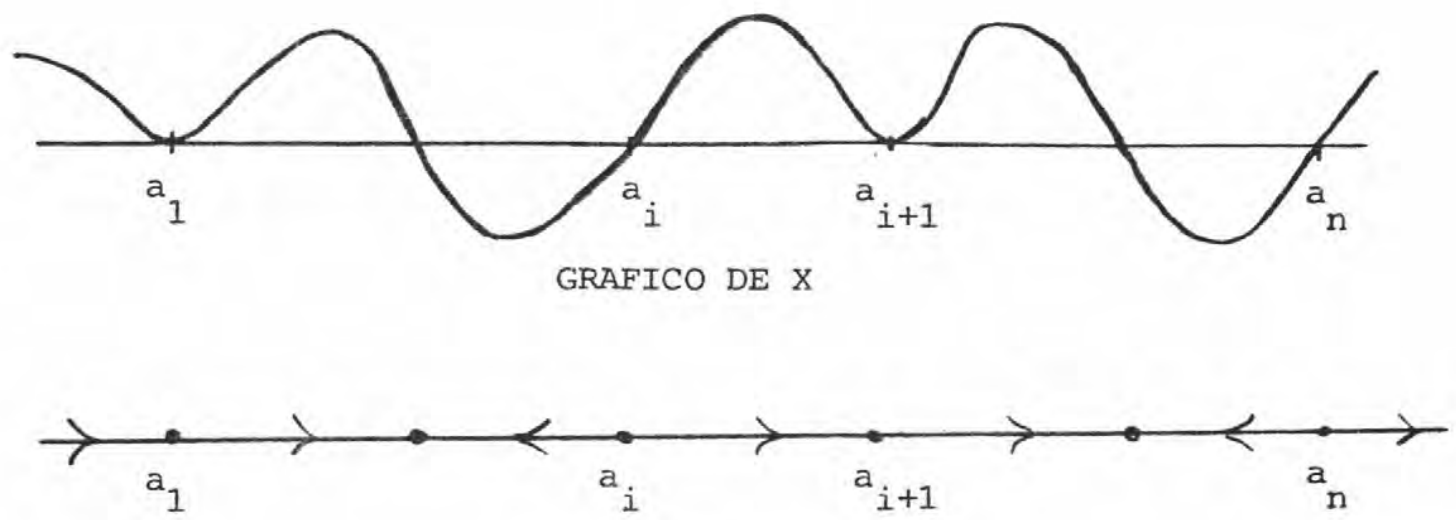

RETRATO DE FASE DE $X$

\section{2.- Campos Simples en el plano real.}

Por campos simples en el plano real, entenderemos siste-

mas reales de la forma

$$
\begin{cases}x_{1}^{\prime}=a_{11} x_{1}+a_{12} x_{2} & \\ x_{2}^{\prime}=a_{21} x_{1}+a_{22} x_{2} & a_{i j} \in \mathbb{R}\end{cases}
$$

y $\quad a_{11} a_{22}-a_{12} a_{21} \neq 0$

Equivalentemente, es una ecuación lineal homogenea del tipo $x^{\prime}=A x, A=\left(\begin{array}{ll}a_{11} & a_{12} \\ a_{21} & a_{22}\end{array}\right)$, $\operatorname{det} A \neq 0$

$\operatorname{det} A \neq 0 \Longrightarrow\{0\} \quad C \mathbb{R}^{2}$ es el único punto singular, es decir el ori gen es el único punto fijo del flujo lineal $\rho(t, x)=e^{t A} x$

Los valores propios de A son

$$
\lambda_{1}, \lambda_{2}=\frac{\operatorname{Traza} A \pm \sqrt{(\operatorname{Traza} A)^{2}-4 \operatorname{det} A}}{2}
$$


Caso a) $\lambda_{1}, \lambda_{2} \in \mathbb{R}, \lambda_{1} \neq \lambda_{2}$ (Necesariamente $\lambda_{1}, \lambda_{2} \neq 0$ )

Sean $v_{1}, v_{2}$ los vectores propios correspondientes a $\lambda_{1}, \lambda_{2}$,

$E_{1}=\left\langle v_{1}\right\rangle$ el subespacio generado por $v_{1}, E_{2}=\left\langle v_{2}\right\rangle$

$\mathbb{R}^{2}=E_{1} \oplus E_{2}$. El flujo en estas coordenadas esta dado por

$$
\rho\left(t,\left(x_{1}, x_{2}\right)\right)=x_{1} e^{\lambda_{1} t} v_{1}+x_{2} e^{\lambda_{2} t} v_{2}
$$

Caso $a_{1}: \lambda_{2}<\lambda_{1}<0$ Nodo atractor (Pozo).

$x \neq 0 \rho(t, x) \rightarrow 0, t \rightarrow+\infty ; \rho(t, x) \rightarrow \infty, t \rightarrow-\infty$

$x=0 \quad \rho(t, 0)=0$

Si $x_{1} \neq 0$, la tangente al flujo tiende a $E_{1}$ cuando $t \rightarrow \infty$ pues

$$
\lim _{t \rightarrow+\infty} \frac{x_{2} e^{\lambda_{2} t}}{x_{1} e^{\lambda_{2} t}}=\frac{x_{2}}{x_{1}} e^{\left(\lambda_{2}-\lambda_{1}\right) t}=0 \text { pues } \lambda_{2}-\lambda_{1}<0
$$

Si $x_{1}=0,\{\rho(t, x)\}$ son las semi-rectas de $E_{2}$

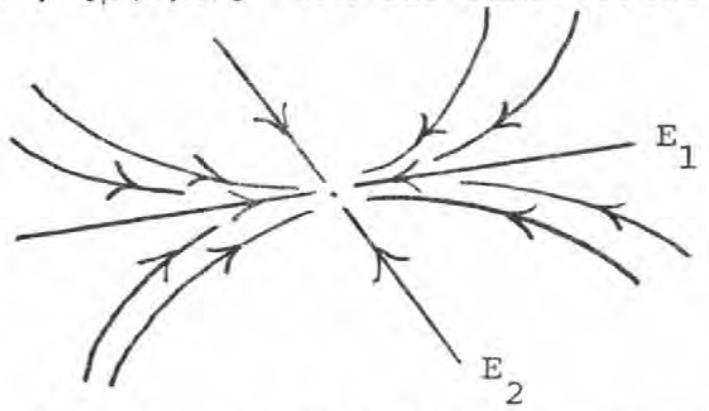

Caso $a_{2}: \lambda_{2}>\lambda_{1}>0$ Nodo repulsor (Fuente) Análisis similar.

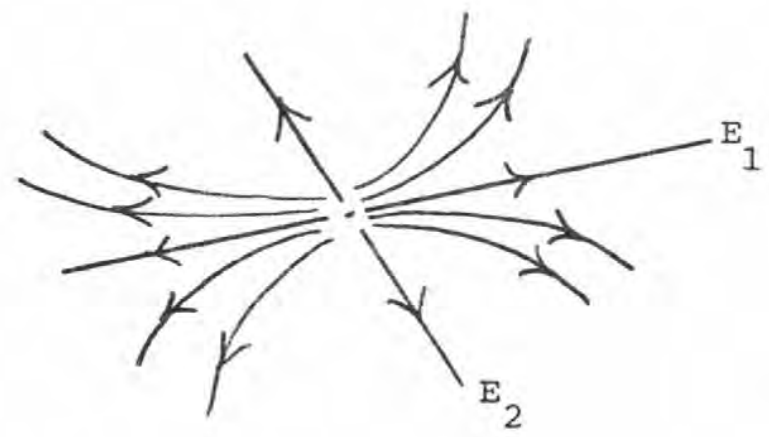


Caso $a_{3}: \lambda_{2}>0>\lambda_{1}$ Silla

Análisis similar

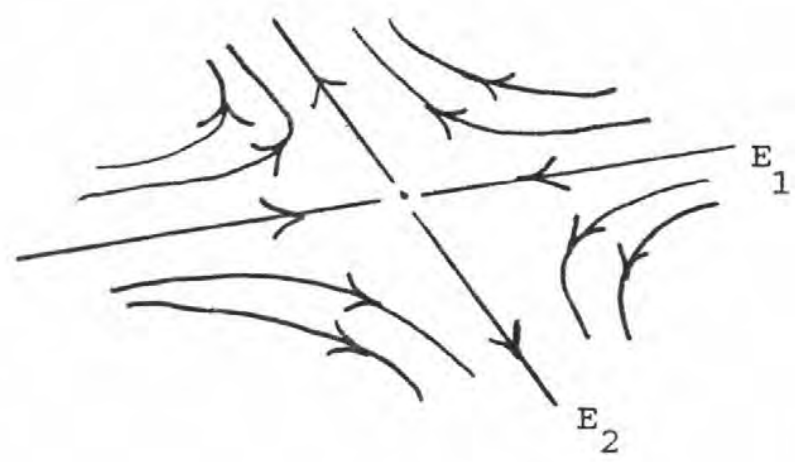

Caso b) $\quad \lambda_{1}, \lambda_{2} \in \mathbb{C}, \lambda_{1}=\alpha+i \beta, \lambda_{2}=\alpha-i \beta$ con $\beta \neq 0$

En este caso $\rho(t, x)=x_{1} p_{1}(t)+x_{2} p_{2}(t)$ donde

$\rho_{1}(t)=e^{\alpha t}\left[\cos \beta t v_{1}-\operatorname{sen} \beta t v_{2}\right]$

$\rho_{2}(t)=e^{\alpha t}\left[\operatorname{sen} \beta t v_{1}+\cos \beta t v_{2}\right]$

Si escribimos $\mathrm{x}$ en coordenadas polares tenemos $\mathrm{x}_{1}=\delta \cos \mathrm{w}$ $\mathrm{x}_{2}=\delta$ sen $\mathrm{w}$. Entonces

$\rho(t, x)=e^{\alpha t} \delta\left[\cos (w-\beta t) v_{1}+\operatorname{sen}(w-\beta t) v_{2}\right]$

Caso $b_{1}: \alpha=0$, Centro

$\forall x \neq 0, \rho(t, x)$ es una elipse. El signo de $\beta$ da la orientación respecto de $t$

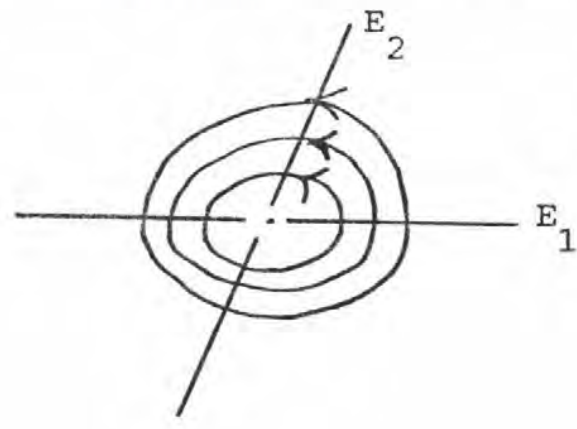


Caso $\mathrm{b}_{2}: \quad \alpha<0$, Foco atractor

||$\rho(t, x) \| \rightarrow 0 ; t \rightarrow \infty y w-\beta t \rightarrow+\infty$ si $\beta<0$

y $w-\beta t \rightarrow-\infty$ si $\beta>0$. Luego las órbitas tienden al origen espiralando, pues $w-\beta t$ mide el ángulo entre $\rho(t)$ con $E_{1}$

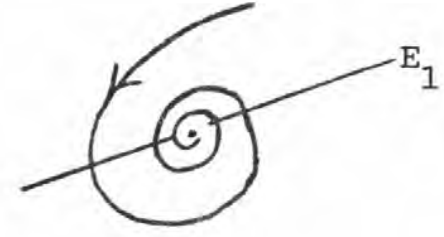

$\beta<0$

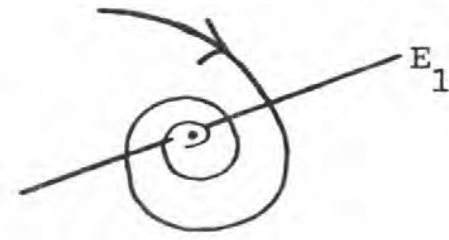

$\beta>0$

Caso $\mathrm{b}_{3}: \alpha>0$ Foco repulsor

Análisis similar

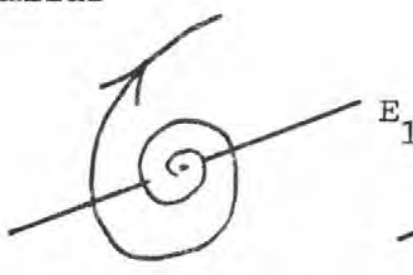

$B>0$

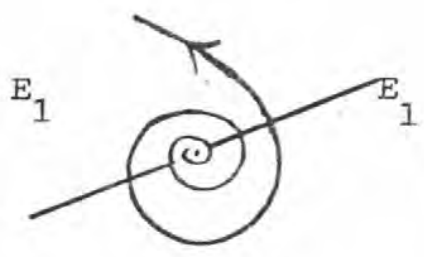

$B<0$

Caso c) $\lambda_{1}=\lambda_{2}=\lambda \neq 0,(\lambda \in \mathbb{R})$ Nodos impropios

Caso $c_{1}$ : Nucelo $A-\lambda I$ es bidimensional. Es decir $\lambda$ tiene vectores propios $\mathrm{v}_{1}, \mathrm{v}_{2}$ linealmente independientes $\rho(t, x)=e^{\lambda t}\left(x_{1} v_{1}+x_{2} v_{2}\right)$. Entonces

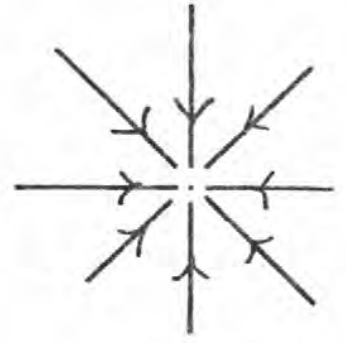

$\lambda<0$

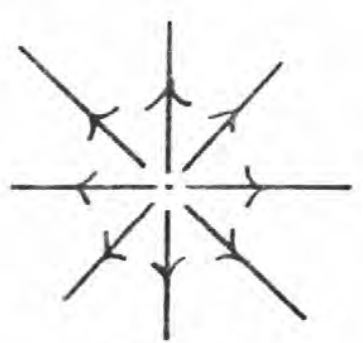

$\lambda>0$ 
Caso $\mathrm{c}_{2}$ : Nucleo $\mathrm{A}-\lambda \mathrm{I}$ es unidimensional. Supongamoslo igual a $\mathrm{E}_{1} \mathrm{Y}$ sea $\mathrm{v}$ un generador de $\mathrm{E}_{1}$. Sea $\mathrm{w}$ un vector no colineal con $\mathrm{v}$. Entonces

$$
\begin{aligned}
& A v=\lambda v \\
& A w=\mu w+\alpha v, \mu, \alpha \in \mathbb{R}, \alpha \neq 0
\end{aligned}
$$

lo que implica que la matriz del operador $\mathrm{x} \rightarrow \mathrm{Ax}$ en la base $\{\mathrm{v}, \mathrm{w}\}$ es

$$
\left(\begin{array}{ll}
\lambda & \alpha \\
0 & \mu
\end{array}\right) \quad, \alpha \neq 0
$$

Los valores propios de esta matriz son $\lambda$ y $\mu$. $\mathrm{Y}$ como los valores propios no dependen de la base $\lambda=\mu$ por hipótesis.

Definamos $v_{1}=\alpha_{v}$

$$
\mathrm{v}_{2}=\mathrm{w}
$$

En esta base $A v_{1}=\lambda v_{1}, A v_{2}=\lambda v_{2}+v_{1} y$ el flujo es

$\rho(t, x)=e^{\lambda t}\left[\left(x_{1}+t x_{2}\right) v_{1}+x_{2} v_{2}\right]$

$x=0, \quad \rho(t, 0)=0$

$x \neq 0 \operatorname{con} x_{2}=0,\{\rho(t, x)\}$ son las semi-rectas de $E_{1}$

$x \neq 0$ con $x_{2} \neq 0$, la tangente al flujo tiende a $E_{1}$ cuando

$t \rightarrow \pm \infty$, pues

$\lim _{t \rightarrow \pm \infty} \frac{x_{2} e^{\lambda t}}{\left(x_{1}+t x_{2}\right) e^{\lambda t}}=\lim _{t \rightarrow \pm \infty} \frac{1}{\frac{x_{1}}{x_{2}}+t}=0$

Además si $x \neq 0, \lim _{t \rightarrow+\infty} \rho(t, x)=0$ si $\lambda<0$

$$
\lim _{t \rightarrow-\infty} \rho(t, x)=0 \text { si } \lambda>0
$$

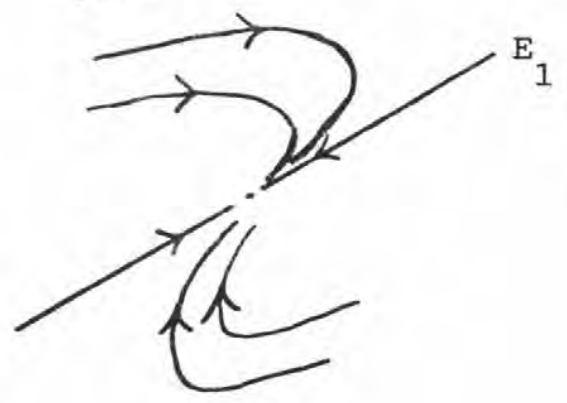

$\lambda<0$

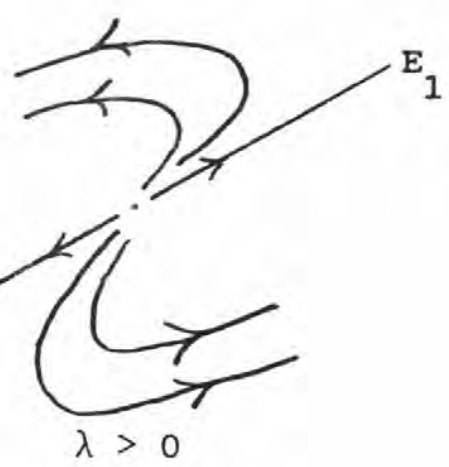




\section{3.- Otro ejemplo de Retrato de Fase.}

$$
\text { Sea } x: \mathbb{R}^{2} \rightarrow \mathbb{R}^{2},(\mathrm{x}, \mathrm{y}) \rightarrow\left(\mathrm{x},-\mathrm{y}+\mathrm{x}^{3}\right)
$$

el flujo de $\mathrm{X}$ está dado por

$\rho(t,(x, y))=\left(x e^{t},\left(y-\frac{x^{3}}{4}\right) e^{-t}+\frac{x^{3}}{4} e^{3 t}\right)$

Sea $\psi(t, p)$ el flujo de la silla $Y(x, y)=(x,-y)$ del ejemplo anterior Entonces la aplicación

$h: \mathbb{R}^{2} \rightarrow \mathbb{R}^{2}$ definida por $(x, y) \rightarrow\left(x, y+\frac{x^{3}}{4}\right)$

satisface $h(\psi(t, p))=\rho(t, h(p))$

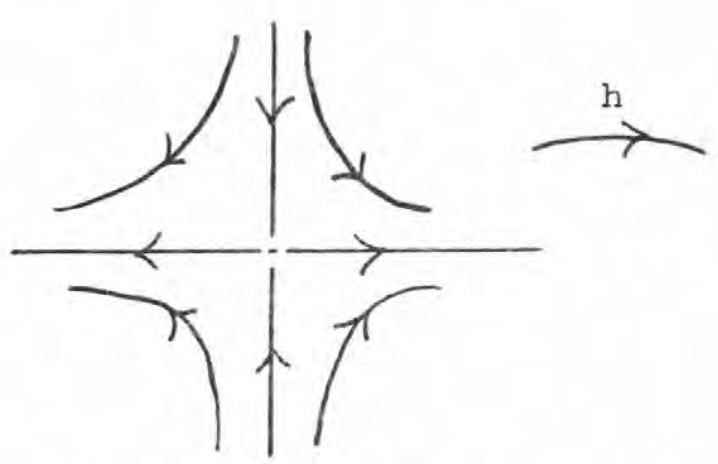

Retrato de Fase de $y$

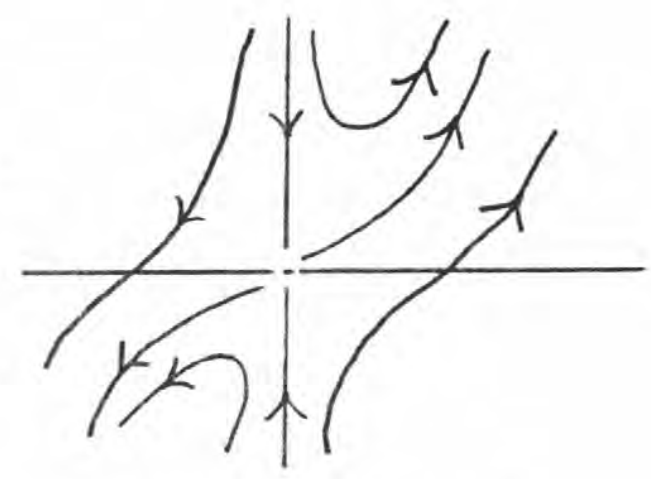

Retrato de Fase de X

Este ejemplo nos motiva al siguiente Capítulo.

\section{§.4. EQUTVALENCIA Y CONJUGACION DE CAMPOS VECTORLALES.}

4.1. Sean $X_{1}: U_{1} C \mathbb{R}^{n} \rightarrow \mathbb{R}^{n} ; X_{2}: U_{2} C \mathbb{R}^{n} \rightarrow \mathbb{R}^{n}$ dos campos vectoriales en $\mathbb{R}^{n} \cdot x_{1}$ se dice topológicamente equivalente a $x_{2}$ cuando existe un homeomorfismo $\mathrm{h}: \mathrm{U}_{1} \rightarrow \mathrm{U}_{2}$ que lleva órbitas de $\mathrm{x}_{1}$ en órbitas de $x_{2}$ preservando orientación.

Es decir; si $p \in U_{1}$ y $\gamma_{1}(p)$ es la órbita orientada de $x_{1}$ pasando por $p$, entonces $h\left(\gamma_{1}(p)\right)$ es la órbita orientada de $x_{2}$ pasando por $h(p), \gamma_{2}(h(p))$. 
Mas preciso; $h\left(\rho_{1}(t, p)\right)=\rho_{2}\left(t^{\prime}, h(p)\right)$ con $t \cdot t^{\prime}>0$ donde $\rho_{1}$ es el flujo de $x_{1}, y \rho_{2}$ el flujo de $x_{2}$.

$\mathrm{Si} h$ es un difeomorfismo de clase $C^{Y}$, los campos se dicen C $C^{x}$-equivalentes. $\left(C^{\circ}\right.$-equivalentes $=$ Topológicamente equivalentes $)$.

Estas definiciones establecen relaciones de equivalencias entre campos definidos en abiertos de $\mathbb{R}^{\mathrm{n}}$.

El homeomorfismo h se llama una equivalencia topológica y el $C^{r}$-difeomorfismo se llama una $C^{r}$-equivalencia.

4.2. $x_{1}$ se dice topológicamente conjugado a $x_{2}$ cuando existe $\mathrm{h}: \mathrm{U}_{1} \rightarrow \mathrm{U}_{2}$ homeomorfismo tal que

$$
h\left(\rho_{1}(t, x)\right)=\rho_{2}(t, h(x)) \forall(t, x) \in D_{1} \text { dominio de } \rho_{1}
$$

Si h es un difeomorfismo de clase $C^{x}$, los campos se dicen

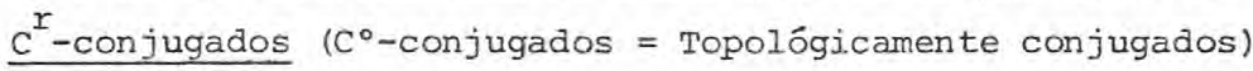
En este caso $I_{x}^{1}=I_{h(x)}^{2}$ (el sobreíndice indica el campo).

El homeomorfismo h se llama una conjugación topológica y el difeo morfismo de clase $C^{r}$ se llama una $C^{r}$-conjugación.

4.3. Observemos que las relaciones de conjugación son de equivalencia entre campos definidos en abiertos de $\mathbb{R}^{n}$.

Toda conjugación es una equivalencia.

Toda equivalencia lleva puntos singulares en puntos singulares y órbitas periódicas en órbitas periódicas.

Si h es una conjugación el período de las órbitas también es pre servado. 


\subsection{Ejemplos:}

a) En el ejemplo 3 de 3.4 . h es una $C^{\infty}$-conjugación.

b) Sean $x^{\prime}=A x \operatorname{con} A=\left(\begin{array}{cc}0 & a \\ -a & 0\end{array}\right) \quad a>0$

$$
x^{\prime}=B x \operatorname{con} B=\left(\begin{array}{cc}
0 & b \\
-b & 0
\end{array}\right) \quad b>0
$$

Estos campos lineales son centros cuyas órbitas periódicas tienen período $\frac{2 \pi}{\mathrm{a}}$ y $\frac{2 \pi}{\mathrm{b}}$

Si $\mathrm{a} \neq \mathrm{b}$ estos campos no son conjugados.

La identidad de $\mathbb{R}^{2}$ define una $C^{\infty}$-equivalencia entre estos campos.

4.5. Lema de Caracterización de conjugaciones diferenciales.

Sean $x_{1}: U_{1} C \mathbb{R}^{n} \rightarrow \mathbb{R}^{n}, x_{2}: U_{2} C \mathbb{R}^{n} \rightarrow \mathbb{R}^{n}$ campos de clase $\mathrm{C}^{\mathrm{x}} \mathrm{y} \mathrm{h}: \mathrm{U}_{1} \rightarrow \mathrm{U}_{2}$ un difeomorfismo de clase $\mathrm{C}^{\mathrm{r}}$. Entonces $\mathrm{h}$ es una con jugación entre $\mathrm{x}_{1}$ y $\mathrm{x}_{2}$ si y sólo si

$D h(p) x_{1}(p)=x_{2}(h(p)) \quad \forall p \in U_{1} \cdots(*)$

Demostración:

$\Longleftarrow$, sea $p \in U_{1}$, definamos $\psi(t)=h\left(p_{1}(t, p)\right), t \in I_{p}^{1}$ $\psi$ es solución de $x^{\prime}=x_{2}(x), x(0)=h(p)$, pues;

$\psi^{\prime}(t)=\operatorname{Dh}\left(\rho_{1}(t, p)\right) \frac{d}{d t} \rho_{1}(t, p)=\operatorname{Dh}\left(\rho_{1}(t, p)\right) x_{1}\left(\rho_{1}(t, p)\right)$

$=x_{2}\left(h\left(\rho_{1}(t, p)\right)\right)=x_{2}(\psi(t))$ y $\psi(0)=h(p)$

Pero $\rho_{2}(t, h(p))$ es solución de $x^{\prime}=x_{2}(x), x(0)=h(p)$

entonces por unicidad $h\left(\rho_{1}(t, p)=\rho_{2}(t, h(p))\right.$

$\Longrightarrow$ Derivemos respecto de $t$ en $t=0 h\left(\rho_{1}(t, p)\right)=\rho_{2}(t, h(p))$

$\left.\operatorname{Dh}\left(\rho_{1}(0, p)\right) \frac{d}{d t} \rho_{1}(t, p)\right|_{t=0}=\left.\frac{d}{d t} \rho_{2}(t, h(p))\right|_{t=0}$

es decir Dh $(p) x_{1}(p)=x_{2}(h(p))$. 
4.6. Sea $X: U \subseteq \mathbb{R}^{n} \rightarrow \mathbb{R}^{n}$ un campo de clase $c^{x}, x \geqslant 1$ y sea $A \subseteq \mathbb{R}^{n-1}$ un abierto. Llamaremos sección transversal local de $\mathrm{X}$ a una aplicación de clase $C^{X}, f: A \rightarrow U$ tal que $\forall$ a $\in A$ $\operatorname{df}(\mathrm{a})\left(\mathbb{R}^{\mathrm{n}-1}\right)$ y $x(f(a))$ general $\mathbb{R}^{\mathrm{n}}$.

Sea $\sum=f(A)$. si $f: A \rightarrow \sum$ es un homeomorfismo $\sum$ se dice una sección transversal de $\mathrm{X}$

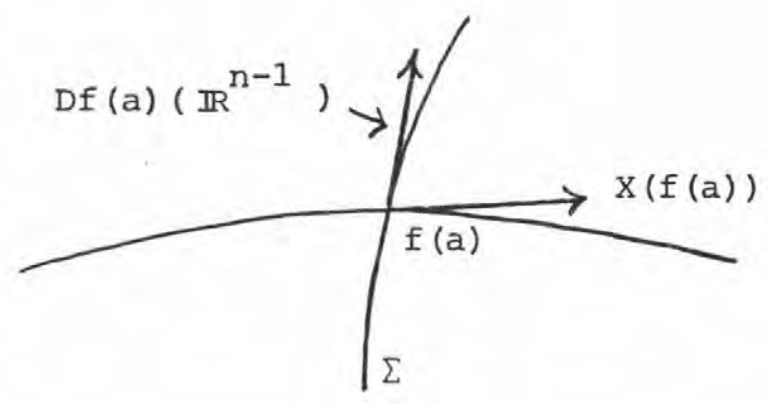

4.7. Sea $p \in U$ no singular $y \quad\left\{v_{1}, \ldots, v_{n-1}, x(p)\right\}$ base de $\mathbb{R}^{n}$. Sea $B(0, \delta)$ bola en $\mathbb{R}^{n-1}$. Entonces para $\delta$ suficientemente pequenิo

$$
\begin{aligned}
f: B(0, \delta) & \subset \mathbb{R}^{n-1} \rightarrow U \subseteq \mathbb{R}^{n} \\
\left(x_{1}, \ldots, x_{n-1}\right) & \rightarrow p+\sum_{i-1}^{n-1} x_{i} v_{i}
\end{aligned}
$$

es una sección transversal local de $x$ en $p$.

\subsection{Teorema del Flujo Tubular.}

Sea $p \in U$, no singular del campo $x: u \subseteq \mathbb{R}^{n} \rightarrow \mathbb{R}^{n}$ de clase $\mathrm{C}^{\mathrm{k}}$ y $\mathrm{f}: \mathrm{A} \rightarrow\left[\right.$ sección transversal local de $\mathrm{x}$ de clase $\mathrm{C}^{\mathrm{k}}$ con $f(0)=p$.

Entonces existe $\mathrm{V}$ vecindad de $\mathrm{p}$ en $\mathrm{U}$ y un difeomorfismo $\mathrm{h}: \mathrm{V} \rightarrow(-\varepsilon, \varepsilon) \times \mathrm{B}$ de clase $\mathrm{C}^{\mathrm{k}}$ con $\varepsilon>0$ y $\mathrm{B}$ una bola abierta de $\mathbb{R}^{\mathrm{n}-1}$ con centro en $0=f^{-1}(p)$ tal que 
a) $\mathrm{h}([\cap \mathrm{V})=\{0\} \times \mathrm{B}$

b) Si y es el campo constante $(1,0, \ldots, 0)$ en $\mathbb{R}^{n}$, entonces $h$ es una $C^{r}$-conjugación entre $\left.\mathrm{x}\right|_{V}$ con $\left.\mathrm{Y}\right|_{(-\varepsilon, \varepsilon) \times B}$

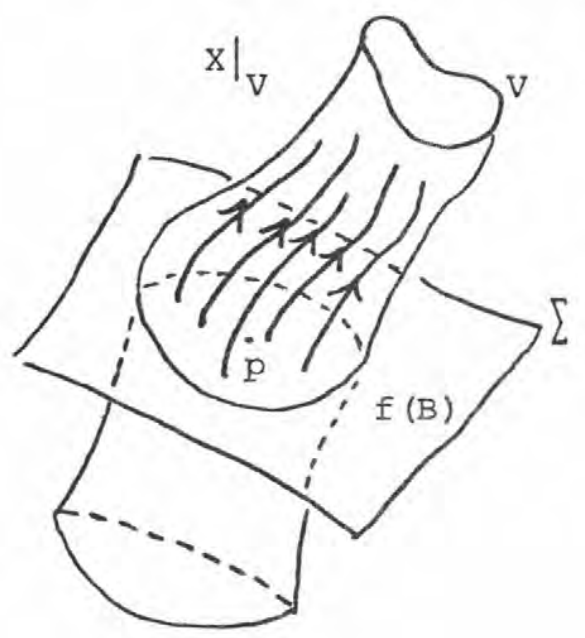

Demostración:
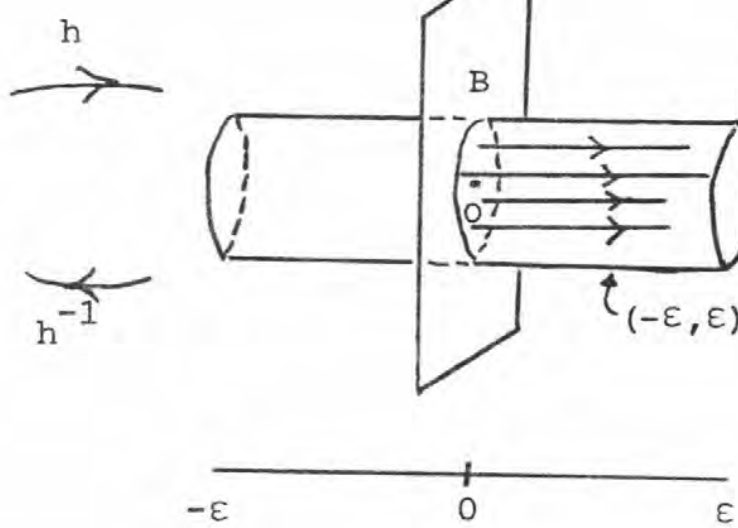

Sea $\rho: D \rightarrow U$ el flujo de $x$. Definamos

$F:\left\{(t, u) \in \mathbb{R} \times \mathbb{R}^{n-1} /(t, f(u)) \in D\right\} \quad \rightarrow \quad$ u por

$F(t, u)=\rho(t, f(u)) . \quad F$ aplica las rectas $l_{u}=\{(t, u) / t \in \mathbb{R}\}$ de $\mathbb{I}^{n}$ en las curvas integrales $\rho(t, f(u))$ de $x$.
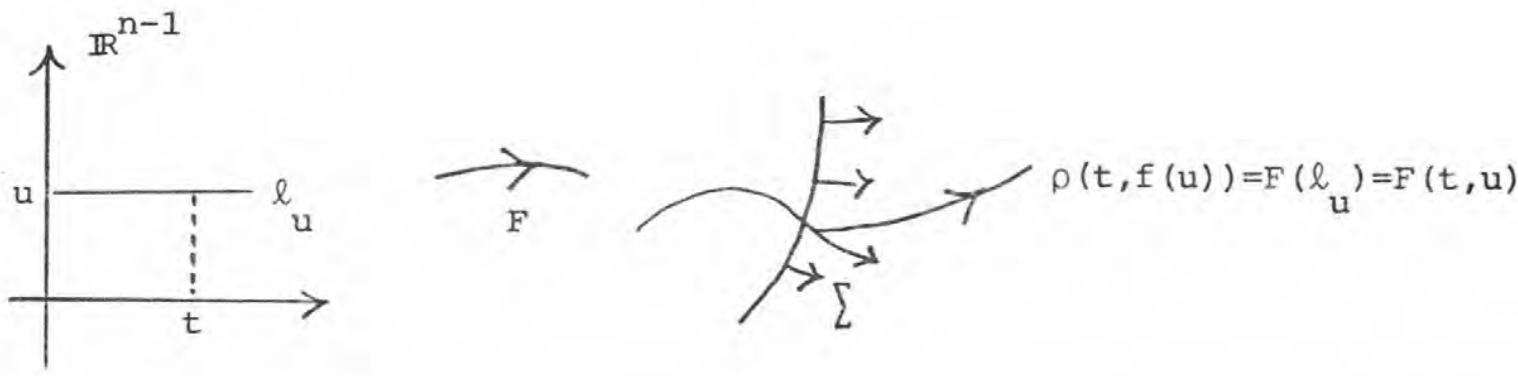
Supongamos que $F$ es un difeomorfismo local en $(0,0)$ e $\mathbb{R} \times \mathbb{R}^{n-1}$ entonces existe $\varepsilon>0$ y una bola $B$ con centro en 0 en $\mathbb{R}^{n-1}$ tal que $\left.F\right|_{(-\varepsilon, \varepsilon) \times B}$ es difeomorfismo sobre $V=F((-\varepsilon, \varepsilon) \times B)$ sea $h=\left(\left.F\right|_{(-\varepsilon, \varepsilon) \times B}\right)^{-1}$. Entonces

a) $h\left(\sum \cap \quad v\right)=\{0\} \times B$ pues $F(0, u)=\rho(0, f(u))=f(u)$ $\forall u \in B \Rightarrow F^{-1}(f(u))=(0, u)$

b) $\mathrm{h}^{-1}$ conjuga $\mathrm{Y}$ con $\mathrm{X}$ pues usando Lema 4.5. tenemos

$$
\begin{aligned}
\operatorname{Dh}^{-1}(t, u) Y(t, u) & =D F(t, u)(1,0, \ldots, 0)=D_{1} F(t, u) \\
& =D_{1} \rho(t, f(u))=X(\rho(t, f(u))=X(F(t, u)) \\
& =X\left(h^{-1}(t, u)\right) \quad \forall(t, u) \in(-\varepsilon, \varepsilon) \times B
\end{aligned}
$$

Nos resta ver que $F$ es un difeomorfismo local. Por el Teorema de la función inversa es suficiente probar que DF(0) es un isomorfismo.

$D_{1} F(0)=\left.\frac{d}{d t} \rho(t, f(0))\right|_{t=0}=x(\rho(0, p))=x(p)$

$D_{j} F(0)=D_{j-1} f(0) \quad \forall j=2, \ldots, n$ pues $\rho(0, f(u))=f(u) \quad \forall u \in A$ Luego los vectores $D_{j} F(0), j=1, \ldots, n$ generan $\mathbb{R}^{n}$, es decir $\mathrm{DF}(0)$ es un isomorfismo.

4.9. Corolario: Sea $\sum$ sección transversal de $x$. Entonces $\forall p \in \Sigma$ existen $\varepsilon>0$, una vecindad $v$ de $p$ en $\mathbb{R}^{n} y$ una función $\tau: V \rightarrow \mathbb{R}$ de clase $C^{k}$ tal que:

a) $\tau(V \cap \Sigma)=0$

b) $\forall \mathrm{q} \in \mathrm{V}$, la curva integral $\rho(t, q)$ de $\left.x\right|_{V}$ está definida $\mathrm{y}$ es biuní voca en $J_{q}=(-\varepsilon+\tau(q), \varepsilon+\tau(q))$

c) $\xi(q)=\rho(\tau(q), q)$ e $\Sigma$ es el único punto donde $\left.\rho(t, q)\right|_{J_{q}} \cap \Sigma$. En particular $q \in \sum \Omega V \Longleftrightarrow \tau(q)=0$ 
d) $\xi: \mathrm{V} \rightarrow \sum$ es de clase $\mathrm{C}^{\mathrm{k}}$ y $\mathrm{D} \xi(\mathrm{q})$ es sobrejectiva $\forall \mathrm{q} \in \mathrm{V}$ En este Corolario se definen 2 aplicaciones fundamentales: $\tau(q)$ mide el tiempo que queda para llegar a $\sum$ por el flujo de X (o inide el tiempo que pasó desde que el flujo pasó por $\Sigma$ ) y $\xi(q)$ que es el punto por donde pasa el flujo de $q$ por $\Sigma$

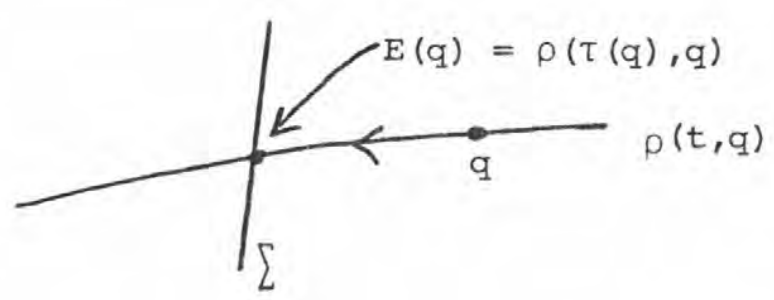

Usando el Teorema 4.8 intente una demostración de 4.9.

4.10. Observemos que al teorema del flujo tubular es solo local. El siguiente ejemplo muestra que no siempre un campo sin singularidades se puede trivializar.

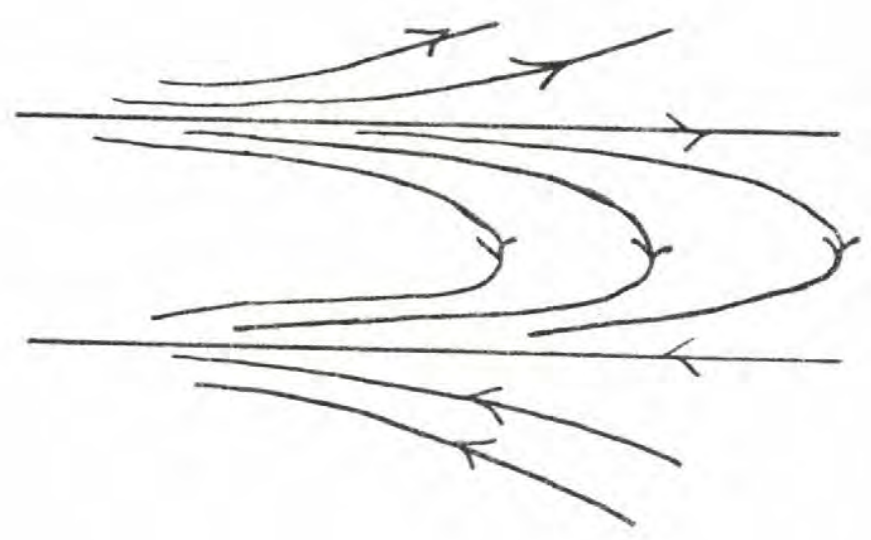




\section{§.5. ESTRUCTURA LOCAL DE LOS PUNTOS SINGULARES HIPERBOIICOS.}

El teorema del flujo tubular describe satisfactoriamente la conducta cualitativa de un campo en torno de un punto regular. Vimos también en el caso de $\mathbb{R}^{2}$ la estructura en torno de un punto singular de campos lineales simples. Ahora estudiaremos la clase más amplia de campos en los cuales persiste la conducta en torno de una singula ridad dada por la parte lineal del campo.

5.1. Sea $X: U \subseteq \mathbb{R}^{n} \rightarrow \mathbb{R}^{n}, p \in U$, punto singular ed $X(p)=0$

$\mathrm{x}$ de clase $\mathrm{C}^{\mathrm{k}}, \mathrm{k} \geqslant 1$. $\mathrm{p}$ se llama Hiperbólico si todos los autovalores de DX $(p)$ tienen parte real diferente de cero.

5.2. Esta definición no depende de la clase de conjugación local c ${ }^{2}$ de $X$ en $p$. Pues si $X$ e $Y$ son campos de clase $C^{k}, k \geqslant 2 y h$ una $\mathrm{C}_{2}$-conjugación entre $\mathrm{X}$ e $\mathrm{Y}$ en torno de la singularidad $\mathrm{p}$ de $\mathrm{X} \mathrm{Y}$ $q=h(p)$ de $Y$.

Por el lema de caracterización 4.5 se tiene $\mathrm{Y}=\mathrm{Dh} \circ \mathrm{h}^{-1}$. $\mathrm{x} \circ \mathrm{h}^{-1}$ es decir

$$
\begin{aligned}
D Y(q)= & D^{2} h\left(h^{-1}(q)\right) D h^{-1}(q) X\left(h^{-1}(q)\right) \\
& +D h\left(h^{-1}(q)\right) D X\left(h^{-1}(q)\right) D^{-1}(q) \\
= & 0+D h \text { (p) DX (p) }[D h(p)]^{-1}
\end{aligned}
$$

por lo tanto las matrices $D Y(q), D X(p)$ son similares, es decir tienen los mismos autovalores.

5.3. El número de autovalores de $D X(p)$ que tienen parte real menor que 0 para un campo hiperbólico en p se llama el índice de estabilidad de $\mathrm{x}$ en $\mathrm{p}$. 


\subsection{Teorema;}

Dos sistemas lineales hiperbólicos $\mathrm{x}^{\prime}=\mathrm{Ax}$ y $\mathrm{x}^{\prime}=\mathrm{Bx}$ en $\mathbb{R}^{\mathrm{n}}$ son topologicamente conjugados si y solamente si ambos tienen el mismo Indice de estabilidad.

\section{Demostración:}

Si $\mathrm{S}$ es el Índice de estabilidad, basta demostrar que ambos campos son conjugados al sistema

$$
\left\{\begin{array}{l}
x_{1}^{\prime}=-x_{1}, x_{1} \in \mathbb{R}^{S} \\
x_{2}^{\prime}=x_{2}, x_{2} \in \mathbb{R}^{n-s}
\end{array}\right.
$$

$\mathrm{Y}$ esto resulta por la forma simple que tienen los flujos lineales. Para el recíproco hay que usar el Teorema de la invarianza de la dimensión de Brouwer.

El siguiente resultado, uno de los más relevantes en la teoría cualitativa de las ecuaciones diferenciales generaliza 5.4, es de cir el índice determina la clase de conjugación topológica local para campos hiperbólicos.

\subsection{Teorema de Hartman:}

Sea $X: U \subseteq \mathbb{R}^{n} \rightarrow \mathbb{R}^{n}$ campo vectorial de clase $C^{1}$ en $p$, punto singular hiperbólico. Entonces existen vecindades $V$ de $p$ en U Y W de 0 en $\mathbb{R}^{n}$ tal que $\left.x\right|_{V}$ es topológicamente conjugado a $\left.D x(p)\right|_{W}$.

Luego aplicando Teorema 5.4. podemos clasificar localmente los puntos singulares hiperbólicos. 


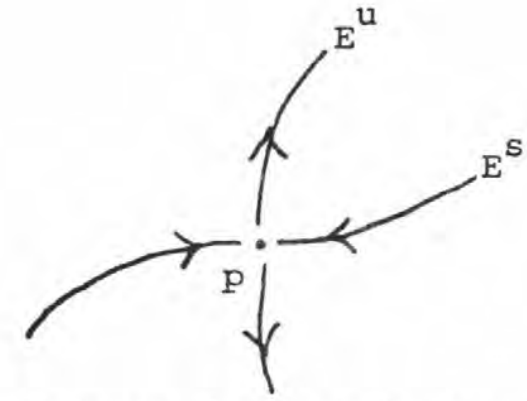

Retrato de Fase de $x$ en $p$

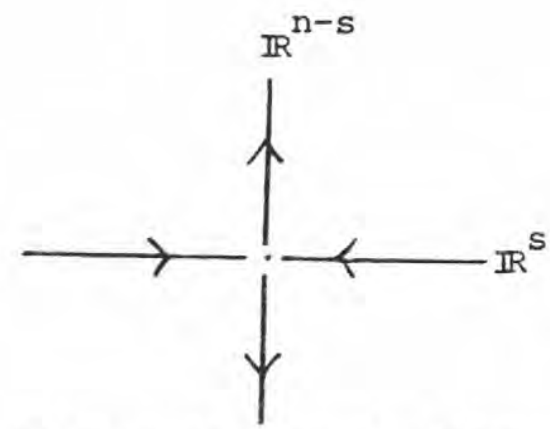

Retrato de Fase de $\mathrm{DX}(\mathrm{p})$ en 0.

La demostración de este Teorema es no trivial y escapa a los objetivos y tiempo! de este cursillo. Una demostración geométrica puede encontrarse en el libro de J. Palis, W. de Melo.

\section{§.6. ESTRUCTURA LOCAL DE ORBITAS PERIODICAS.}

\subsection{La Transformación de Poincaré.}

Sea $\gamma=\left\{\rho(t, p) / 0 \leqslant t \leqslant \tau_{0}\right\}$ una órbita periódica de período $\tau$ del campo $\mathrm{x}$ de clase $C^{k}, k \geqslant 1$ definido en $U \underline{c} \mathbb{R}^{n}$.

Sea $\sum$ una sección transversal a $x$ en $p . \quad \forall q \in \sum$ próximo a $p$ la trayectoria $\rho(t, q)$ esta próxima de $\gamma$ por la continuidad del flujo.

Definimos $\pi(q)$ como el primer punto donde $\rho(t, q)$ interstecta

a $\Sigma$. Sea $\Sigma_{0}=$ Dominio de $\pi$. Obviamente $p$ e $\Sigma_{0} y \pi(p)=p$.

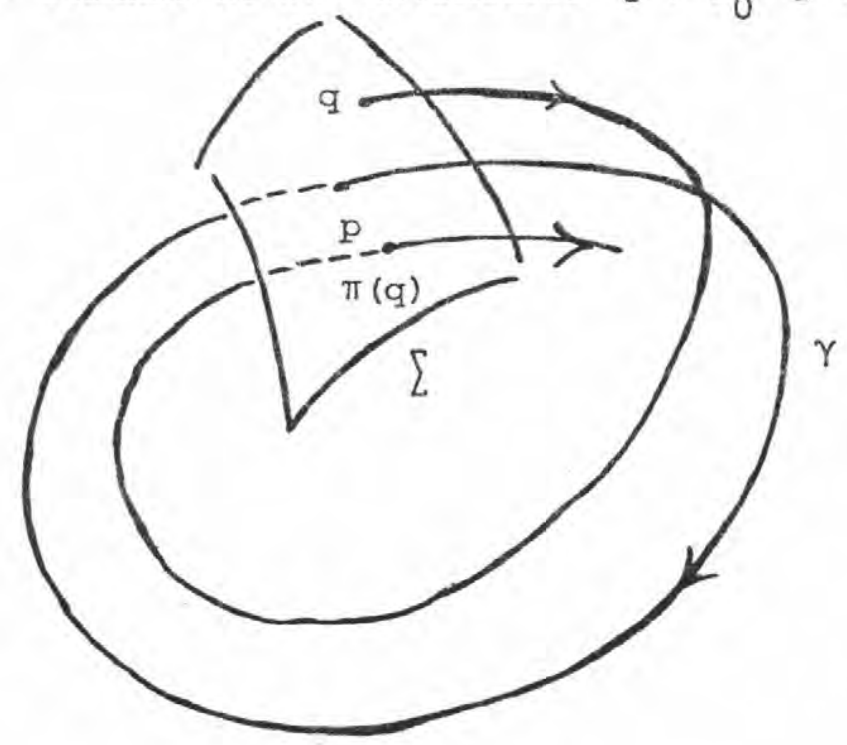


Observemos que las órbitas periódicas de $\mathrm{x}$ vecinas de $\gamma$ corresponden a puntos periódicos de $\pi$, que son los puntos $q$ e $\sum_{0}$ tq $\pi^{n}(q)=q$ para algún $n \in \mathbb{N}$.

El comportamiento asintótico de las órbitas de $\mathrm{x}$ cerca de $\gamma$ es también descrito por $\pi$. Por ejemplo

$$
\lim _{n \rightarrow \infty} \pi^{n}(q)=p \Longrightarrow \lim _{t \rightarrow \infty} d(p(t, q), \gamma)=0
$$

6.2. Una órbita cerrada $\gamma$ se llama un atractor periódico ó $\gamma$ se dice orbitalmente estable cuando $\lim _{t \rightarrow \infty} d(p(t, q), \gamma)=0 \forall q$ en una vecindad de $\gamma$.

6.3. Lema:

$\pi: \Sigma_{0} \rightarrow \Sigma$ es un difeomorfismo de clase $c^{k}$ sobre su imagen $\Sigma_{1}$. Demostración:

Podemos suponer sin perder generalidad que $\sum$ es una hipersuperficie o una subvariedad diferenciable de dimensión (n-1) de $U \subseteq \mathbb{R}^{n}$. Más aun podemos suponer que $\sum$ es un disco de un subespacio vectorial o afin de $\mathbb{R}^{\mathrm{n}}$.

Usaremos el corolario 4.9 para definir formalmente $\pi$.

Sea $\mathrm{V}$ una vecindad de $\mathrm{p}$ dada por el Corolario 4.9 como

$\rho\left(\tau_{0}, p\right)=p$ existe $\Sigma_{0}$ vecindad de $p$ en $\Sigma$ tal que

$\rho\left(\tau_{0}, q\right) \in v \quad \forall q \in \Sigma_{0}$

Sea $\xi: V \rightarrow \Sigma$ definida por 4.9 , entonces

$\pi: \Sigma_{0} \rightarrow \Sigma, q \rightarrow \pi(q)=\xi\left(\rho\left(\tau_{0}, q\right)\right)$

otra expresión equivalente para $\pi$, es $\pi(q)=\rho\left(\tau_{0}+\tau\left(\rho\left(\tau_{0}, q\right)\right), q\right)$ donde $\tau$ está definida en 4.9, $\tau: V \rightarrow \mathbb{R}$ es el tiempo $\tau(x)$ que demora la órbita de $\mathrm{x}$ en $\mathrm{V}$ para interceptar a $\Sigma$. Por teorema de la función implícita $\tau$ es de clase $c^{k}$. Por lo tanto $\pi$ es de clase $c^{k}$. Ia inver sa $\pi-^{1}: \Sigma_{1} \rightarrow \Sigma_{0}$ de $\pi$ está definida tomando el campo $-x$. 


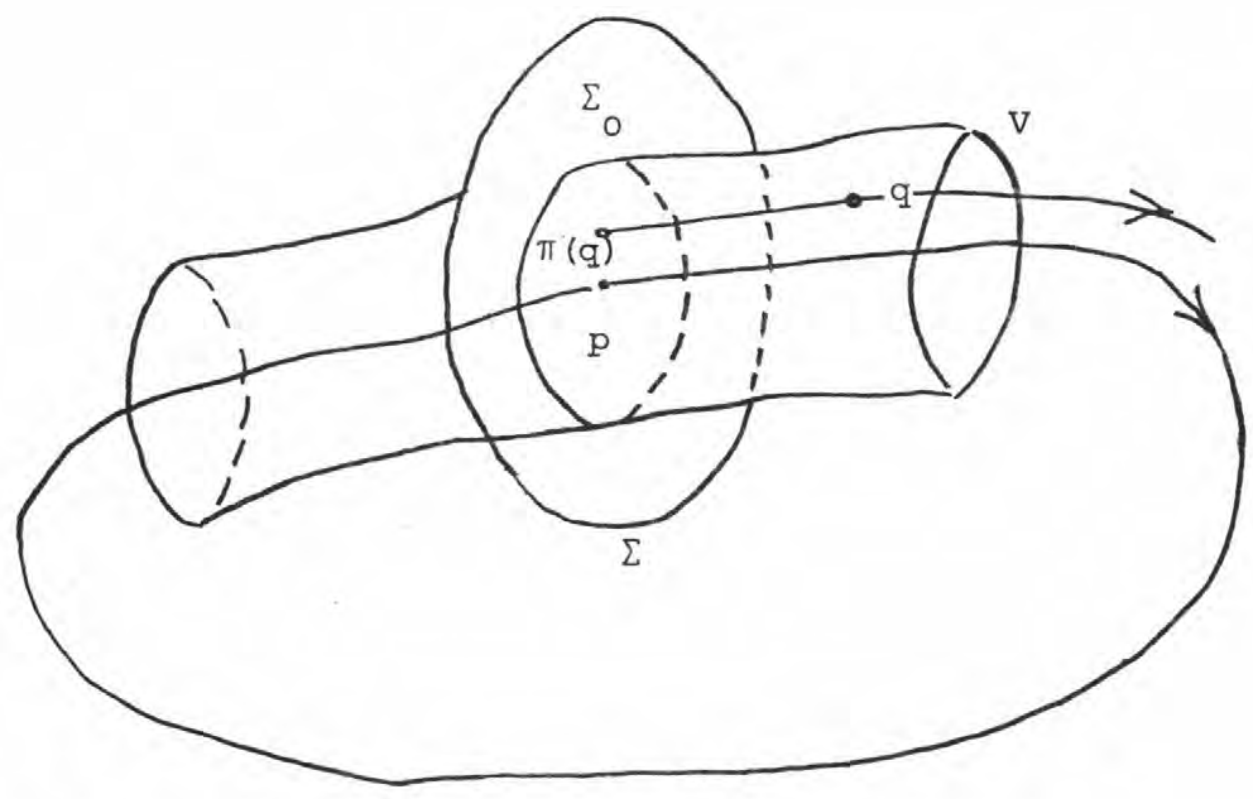

$$
\pi(q)=\xi\left(\rho\left(\tau_{0}, q\right)\right)=\rho\left(\tau_{0}+\tau\left(\rho\left(\tau_{0}, q\right)\right), q\right)
$$

6.4. Ciclos Límites en el plano.

Sea $X: U \subseteq \mathbb{R}^{2} \rightarrow \mathbb{R}^{2}$, $U$ abierto, $x$ de clase $C^{1}$. Una órbita periódica $\gamma$ de $X$ se llama un ciclo límite si existe una vecindad $V$ de $\gamma$ tal que $\gamma$ es la única órbita cerrada de $x$ que intercepta $v$.

Proposición:

Solo existen los siguientes tipos de ciclos límites en el plano.
a) Ciclo estable:
Si $\lim _{t \rightarrow \infty} d(\rho(t, q), \gamma)=0 \quad \forall q \in v$
b) Ciclo inestable: Si $\lim _{t \rightarrow-\infty} d(\rho(t, q), \gamma)=0 \quad \forall q \in V$

c) Ciclo semi-estable: Si $\lim _{t \rightarrow \infty} d(\rho(t, q), \gamma)=0$ para todo

$q \in V \cap$ Exterior de $\gamma y \lim _{t \rightarrow \infty} d(\rho(t, q), \gamma)=0 \forall q \in V \cap$ Interior de $\gamma$ - 10 contrario. 


\section{Demostración:}

Podemos suponer que $\mathrm{V}$ no contiene singularidades de $\mathrm{X}$. Sea $\mathrm{p} \in \gamma \mathrm{y} \sum$ una sección transversal a $\mathrm{X}$ en $\mathrm{p}$.

Sea $\pi: \Sigma_{0} \rightarrow \sum$ transformación de Poincaré. Supongamos que $\Sigma$ está orde nada en el sentido positivo desde el Ext. al Int.

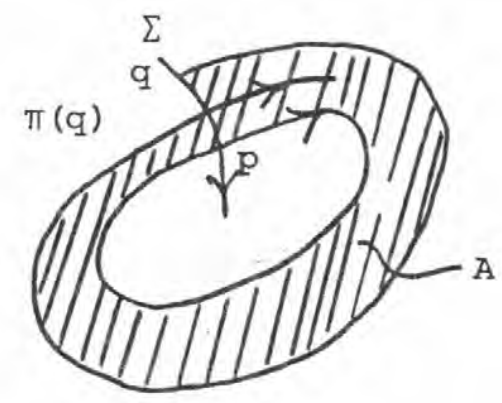

Dado $q \in \Sigma_{0} \cap$ Ext $\gamma$ tenemos $\pi(q)>q \delta \sigma(q)<q$

Supongamos $\pi(q)>q$. Consideremos la región $A$ acotada por $\gamma$, por el arco de trayectoria $\overparen{q \pi(q)}$ y por el segmento $\overline{q \pi(q)} \in \Sigma_{0}$

$A$ es positivamente invariante, es decir, $\forall x \in A, \rho(t, x) \in A$

$\forall t \geqslant 0$. Más aun $\rho(t, x)$ intercepta $\sum$ en una sucesión estrictamente monótona de puntos $x_{n}$ que converge a $p$, es decir

$\lim d(\rho(t, x), \gamma)=0$

$t \rightarrow \infty$

Si $\pi(q)<q$ consideremos el campo $-\mathrm{x}$ y allí se concluye

$\lim _{t \rightarrow \infty} d(\rho(t, x), \gamma)=0 \quad \forall x \in A$

$t \rightarrow \infty$

Io mismo se puede hacer para Int. de $\gamma y$ combinando las posi bilidades resulta a), b) y c).

\subsection{Lema:}

a) $\gamma$ es un ciclo límite si y solo si p es un punto fijo aislado de $\pi$. 
b) $\gamma$ es estable si y solo si $|\pi(x)-p|<|x-p| \forall x \neq p, x \in \Sigma_{0}$
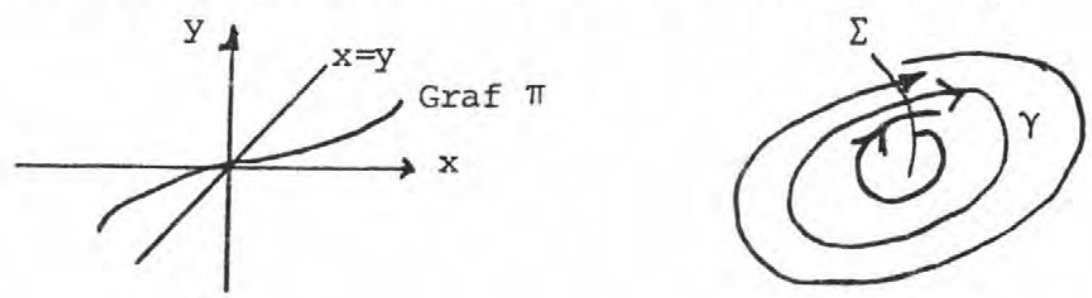

c) $\gamma$ es inestable si y solo si $|\pi(x)-p|>|x-p| \forall x \neq p, x \in \Sigma_{0}$
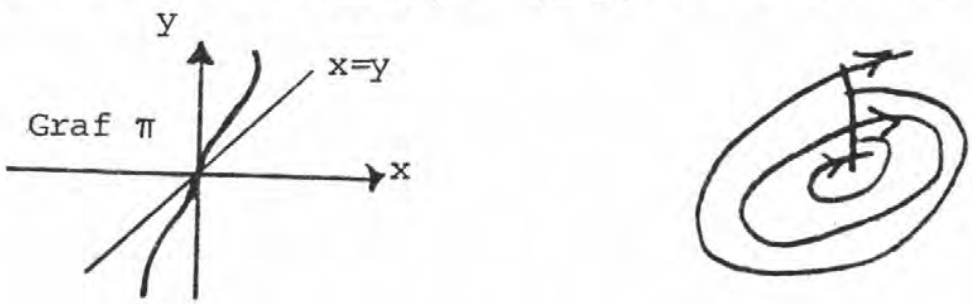

d) $\gamma$ es semi-estable si y solo si $|\pi(x)-p|<|x-p| \forall x \in \sum$ Ext $\gamma$ próximo de $p,|\pi(x)-p|>|x-p| \forall x \in \sum \Omega$ Int $\gamma$, próximo de $p$ бo el contrario
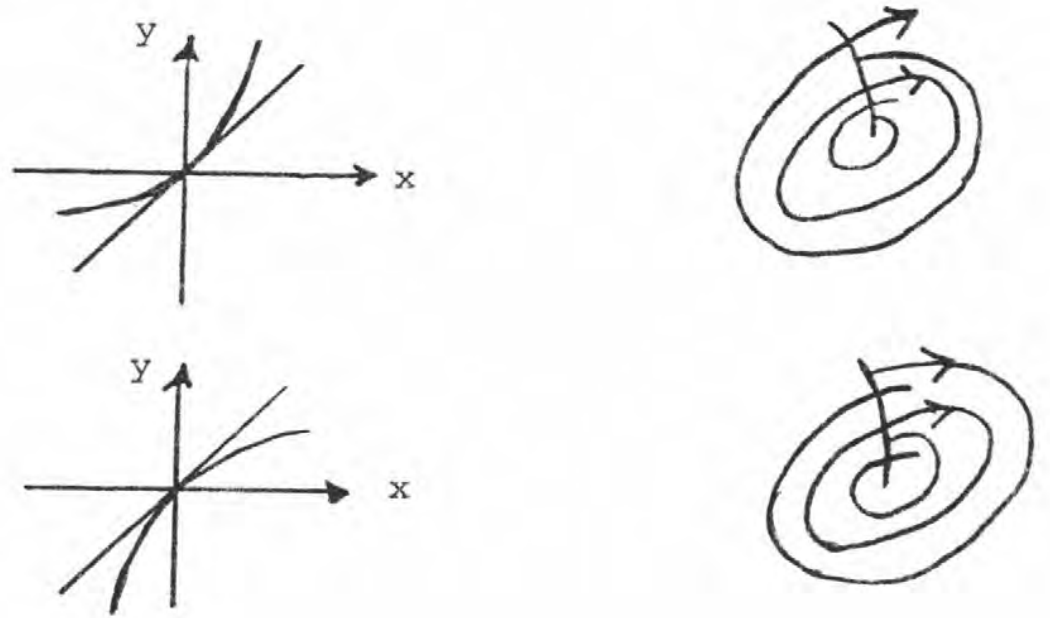

\section{Demostración:}

Obvia. 
6.6. Lema:

a) Si $\pi^{\prime}(\mathrm{p})<1 \Longrightarrow \gamma$ es estable

b) $\operatorname{Si} \pi^{\prime}(\mathrm{p})>1 \Longrightarrow \gamma$ es inestable

Demostración:

Aplicar el Teorema del Valor Medio y usar 6.5. Veremos ahora una condición suficiente para que una óxbita periódica sea un ciclo límite estable.

6.7. Teorema:

$\mathrm{X}: U \underline{\mathrm{C}} \mathbb{R}^{2} \rightarrow \mathbb{R}^{2}$ campo de clase $\mathrm{C}^{1} \cdot \gamma$ una órbita periódica de período $\mathrm{T}$ y $\pi: \Sigma_{0} \rightarrow \sum$ transformación de poincaré en una sección trans versal $\sum$ en $p \in \gamma$. Entonces

$$
\pi^{\prime}(p)=\exp \left[\int_{0}^{T} \operatorname{div} X(\gamma(t)) \operatorname{dt}\right]
$$

donde $\operatorname{div} x(x)=D_{1} X_{1}(x)+D_{2} X_{2}(x)$

En particular:

$$
\begin{aligned}
& \text { Si } \int_{0}^{T} \operatorname{div} x(\gamma(t)) d t<0 \Longrightarrow \gamma \text { es estable } \\
& \operatorname{si} \int_{0}^{T} \operatorname{div} x(\gamma(t)) d t>0 \Longrightarrow \gamma \text { es inestable }
\end{aligned}
$$

Demostración:

Recordemos que cuando tenemos una ecuación diferencial lineal $x^{\prime}=A(t) x y$ si $\phi(t)$ es una matriz cuyas columnas son soluciones, entonces $\forall t \in I, t_{0} \in I$ fijo

$\operatorname{det} \phi(t)=\operatorname{det}\left[\phi\left(t_{0}\right)\right] \exp \int_{t_{0}}^{t} \operatorname{Traza} A(s) d s$ 
Pongamos $A(t)=D X(\gamma(t))$ y sea $\phi(t)$ matriz fundamental de $\mathbf{x}^{\prime}=\mathrm{A}(t) \mathrm{X}$ con $\phi(0)=I$ identidad, entonces

$\operatorname{det} \phi(T)=\exp \int_{0}^{T} \operatorname{div} x(\gamma(t)) d t$

demostremos $\pi^{\prime}(p)=\operatorname{det} \phi(T)$

Como $D_{1} D_{2} \rho(T, p)=D X(\rho(T, p)) D_{2} \rho(T, p)=D X(p) D_{2} \rho(T, p)$

es decir $D_{2} \rho(T, p)$ satisface $x^{\prime}=D X(p) x$, luego por unicidad

$\phi(T)=D_{2} \rho(T, p)$

Notemos que $D_{2} \rho(T, p) \cdot X(p)=X(p)$ pues

$\left.\frac{d}{d t} \rho(T, \rho(t, p))\right|_{t=0}:\left.D_{2} \rho(T, \rho(t, p)) \cdot \frac{d}{d t} \rho(t, p)\right|_{t=0}$

$$
=D_{2} \rho(T, p) X(p) \quad y \text { por otro lado }
$$

$\left.\frac{d}{d t} \rho(T, \rho(t, p))\right|_{t=0}=\left.\frac{d}{d t} \rho(T+t, p)\right|_{t=0}=\left.\frac{d}{d t} \rho(t, p)\right|_{t=0}=x(p)$

Por otro lado sea $g:(-\varepsilon, \varepsilon) \rightarrow \sum$ una parametrización de $\Sigma$ tal que $g(0)=p$.

$B=\left\{x(p), g^{\prime}(0)\right\}$ es una base $\mathbb{R}^{2}$ por definición de $\Sigma$

$y \pi(g(s))=\rho(T+\tau(\rho(T, s)), g(s))$, entonces

$\pi^{\prime}(p) \cdot g^{\prime}(0)=\left.\frac{d}{d s} \pi \circ g(s)\right|_{s=0}=D_{1} \rho(T, p) \cdot a+D_{2} \rho(T, p) \cdot g^{\prime}(0)$

$=a x(p)+D_{2} \rho(T, p) \cdot g^{\prime}(0)$

donde a es $\left.\frac{d}{d s} \tau(\rho(T, g(s)))\right|_{s=0}$. Luego 
$D_{2} \rho(T, p) g^{\prime}(0)=\pi^{\prime}(p) g^{\prime}(0)-a x(p)$ Entonces la matriz de
$D_{2} \rho(T, p)$ en la base $B$ es

$$
\left(\begin{array}{ll}
1 & -a \\
0 & \pi^{\prime}(p)
\end{array}\right)
$$

Luego $\operatorname{det} \phi(T)=\pi^{\prime}(p)$ lo que queríamos.

\section{§.7. FLUJOS LTNEALES EN EL TORO.}

Estudiaremos ahora un campo no hiperbólico. Los flujos de campos vectoriales lineales con valores propios puramente imaginarios conduce al estudio de flujos en Superficies Toroidales.

Consideremos en $\mathbb{R}^{4}$ en siguiente sistema

$$
\text { (1) }\left\{\begin{array}{rl}
x_{1}^{\prime}=-\alpha x_{2} \\
x_{2}^{\prime}=\alpha x_{1} \\
x_{3}^{\prime}=-\beta x_{4} \\
x_{4}^{\prime}=\beta x_{3}
\end{array} \quad \alpha, \beta>0\right.
$$

En coordenadas complejas $z_{1}=x_{1}+i x_{2}, z_{2}=x_{3}+i x_{4}$

(2) $\left\{\begin{array}{l}z_{1}^{\prime}=i \alpha z_{1} \\ z_{2}^{\prime}=i \beta z_{2}\end{array}\right.$

El flujo es $\rho\left(t, z_{1}, z_{2}\right)=\left(z_{1} e^{i \alpha t}, z_{2} e^{i \beta t}\right)$

Sean $r_{1}, r_{2}>0 y \operatorname{sean} z_{1}^{0}, z_{2}^{0} \in \mathbb{C}$ tales que $\left|z_{1}^{0}\right|=r_{1},\left|z_{2}^{0}\right|=r_{2}$

La imagen de la curva $t \rightarrow z_{1}^{0} e^{i a t}$ está contenida en 
$c_{1}=\left\{z \in \mathbb{c} /|z|=r_{1}\right\}$. Analogamente la curva $t \rightarrow z_{2}^{0} e^{i \beta t}$ está contenida en $\mathrm{C}_{2}=\left\{\mathrm{z} \in \mathbb{C} /|\mathrm{z}|=\mathrm{r}_{2}\right\}$

Por lo tanto el Toro $\mathrm{T}_{2}=\mathrm{C}_{1} \times \mathrm{C}_{2}$ de $\mathbb{R}^{4}$ es invariante por el flujo $\rho$.

Las soluciones de (1) que están contenidas en $\mathrm{T}^{2}$ son imagen por la aplicación $R: \mathbb{R}^{2} \rightarrow T^{2},\left(\theta_{1}, \theta_{2}\right) \rightarrow\left(r, e^{2 \pi i \theta_{1}}, r_{2} e^{2 \pi i \theta_{2}}\right)$ de las soluciones del sistema de ecuaciones en $\mathbb{I}^{2}$

$$
\begin{aligned}
& \theta_{1}^{\prime}=\alpha / 2 \pi \\
& \theta_{2}^{\prime}=\beta / 2 \pi
\end{aligned}
$$

Sea $c=1 \times C_{2} C \mathbb{C}^{2} . \quad \forall\left(1, z_{2}^{0}\right) \in C$ la órbita $\rho\left(t, 1, z_{2}^{0}\right)$ inter cepta $C$ en una sucesión de puntos $\left(1, \mathrm{z}_{2}^{(n)}\right)$ dada por

$z_{2}^{(n)}=z_{2}^{0} \quad e^{2 \pi n i \beta / \alpha}, n \in \mathbb{z}$.

En realidad estos puntos son los iterados $\pi^{n}\left(z_{2}^{0}\right)$ por la transformación de Poincaré.

$$
\pi: C \rightarrow C, \pi(z)=z e^{2 \pi i \beta / \alpha}
$$

\subsection{Teorema:}

Si $\beta / \alpha$ es racional, todas las órbitas de (2) contenidas en $\mathrm{T}^{2}$ son periódicas.

Si $\beta / \alpha$ es irracional, ellas son densas en $T^{2}$.

\section{Demostración:}

Sea $\beta / \alpha=p / q$ donde $p y q$ son relativamente primos con $q>0$. Entonces todas las órbitas de $\pi$ tienen período $q$, lo que significa que las órbitas de (2) son periódicas de período $2 \pi / q$

Si $\beta / \alpha$ es irracional. Fijemos $z_{2}^{0} \in C_{2}$ y probemos que la sucesión $\pi^{n}\left(z_{2}^{0}\right)$ es densa en el círculo, lo cual es verdaero pues el subgrupo de $(\mathbb{R},+)$ generado por $\{1, \beta / \alpha\}$ es denso en $\mathbb{R}$. 